\title{
Network Pharmacological Analysis through a Bioinformatics Approach of Novel NSC765600 and NSC765691 Compounds as Potential Inhibitors of CCND1/CDK4/PLK1/CD44 in Cancer Types
}

\author{
Ntlotlang Mokgautsi ${ }^{1,2,+} \mathbb{D}$, Yu-Chi Wang ${ }^{3,+}$, Bashir Lawal 1,2 $\mathbb{D}$, Harshita Khedkar 1,2 ${ }^{\mathbb{D}}$, \\ Maryam Rachmawati Sumitra ${ }^{1,2}$, Alexander T. H. Wu 4,5,6,7,*(D) and Hsu-Shan Huang 1,2,7,8,9,*(D)
}

Citation: Mokgautsi, N.; Wang, Y.-C.; Lawal, B.; Khedkar, H.; Sumitra, M.R.; Wu, A.T.H.; Huang, H.-S. Network Pharmacological Analysis through a Bioinformatics Approach of Novel NSC765600 and NSC765691 Compounds as Potential Inhibitors of CCND1/CDK4/PLK1/CD44 in Cancer Types. Cancers 2021, 13, 2523. https://doi.org/10.3390/

cancers13112523

Academic Editor: Paul Lockman

Received: 29 March 2021

Accepted: 18 May 2021

Published: 21 May 2021

Publisher's Note: MDPI stays neutral with regard to jurisdictional claims in published maps and institutional affiliations.

Copyright: (c) 2021 by the authors. Licensee MDPI, Basel, Switzerland. This article is an open access article distributed under the terms and conditions of the Creative Commons Attribution (CC BY) license (https:// creativecommons.org/licenses/by/ $4.0 /)$.
1 PhD Program for Cancer Molecular Biology and Drug Discovery, College of Medical Science and Technology, Taipei Medical University and Academia Sinica, Taipei 11031, Taiwan; d621108006@tmu.edu.tw (N.M.); d621108004@tmu.edu.tw (B.L.); d621108005@tmu.edu.tw (H.K.); d621109006@tmu.edu.tw (M.R.S.)

2 Graduate Institute for Cancer Biology and Drug Discovery, College of Medical Science and Technology, Taipei Medical University, Taipei 11031, Taiwan

3 Department of Obstetrics and Gynecology, Tri-Service General Hospital, National Defense Medical Center, Taipei 11490, Taiwan; yuchitsgh@mail.ndmctsgh.edu.tw

4 TMU Research Center of Cancer Translational Medicine, Taipei Medical University, Taipei 11031, Taiwan

5 The PhD Program of Translational Medicine, College of Science and Technology, Taipei Medical University, Taipei 11031, Taiwan

6 Clinical Research Center, Taipei Medical University Hospital, Taipei Medical University, Taipei 11031, Taiwan Graduate Institute of Medical Sciences, National Defense Medical Center, Taipei 11490, Taiwan

School of Pharmacy, National Defense Medical Center, Taipei 11490, Taiwan

9 PhD Program in Drug Discovery and Development Industry, College of Pharmacy, Taipei Medical University, Taipei 11031, Taiwan

* Correspondence: chaw1211@tmu.edu.tw (A.T.H.W.); huanghs99@tmu.edu.tw (H.-S.H.)

+ These authors contributed equally to this work.

Simple Summary: Around 14 million new cancer cases, rate are reported annually, with high mortality worldswide, several mechanisms are associated with complexities in cancer, which leads to resistance to current therapeutic interventions in cancer patients. The aim of this study was to identify molecular genes responsible for cancer development, progression and resistances to therapeutic intervention, and also evaluate the potency of our novel compounds NSC7565600 and NSC765691 as potential target for these oncogenes. Using bioinformatics, we successfully identified CCND1/CDK4/PLK1/CD44 as oncogenic signatures, which drives cancer progression and resistance to treatment, and as potential druggable candidates for both NSC7565600 and NSC765691 small molecules. We also showed the antiproliferative and cytotoxic effects of these compounds against a panel of NCI-60 cancer cell lines. This suggests the potential of NSC765600 and NSC765691 compounds to inhibit CCND1/CDK4/PLK1/CD44 expressions in cancer.

Abstract: Cyclin D1 (CCND1) and cyclin-dependent kinase 4 (CDK4) both play significant roles in regulating cell cycle progression, while polo-like kinase 1 (PLK1) regulates cell differentiation and tumor progression, and activates cancer stem cells (CSCs), with the cluster of differentiation 44 (CD44) surface marker mostly being expressed. These oncogenes have emerged as promoters of metastasis in a variety of cancer types. In this study, we employed comprehensive computational and bioinformatics analyses to predict drug targets of our novel small molecules, NSC765600 and NSC765691, respectively derived from diflunisal and fostamatinib. The target prediction tools identified CCND1/CDK4/PLK1/CD44 as target genes for NSC765600 and NSC765691 compounds. Additionally, the results of our in silico molecular docking analysis showed unique ligand-protein interactions with putative binding affinities of NSC765600 and NSC765691 with CCND1/CDK4/PLK1/CD44 oncogenic signaling pathways. Moreover, we used drug-likeness precepts as our guidelines for drug design and development, and found that both compounds passed the drug-likeness criteria of molecular weight, polarity, solubility, saturation, flexibility, and lipophilicity, and also exhibited acceptable 
pharmacokinetic properties. Furthermore, we used development therapeutics program (DTP) algorithms and identified similar fingerprints and mechanisms of NSC765600 and NSC765691 with synthetic compounds and standard anticancer agents in the NCI database. We found that NSC765600 and NSC765691 displayed antiproliferative and cytotoxic effects against a panel of NCI-60 cancer cell lines. Based on these finding, NSC765600 and NSC765691 exhibited satisfactory levels of safety with regard to toxicity, and met all of the required criteria for drug-likeness precepts. Currently, further in vitro and in vivo investigations in tumor-bearing mice are in progress to study the potential treatment efficacies of the novel NSC765600 and NSC765691 small molecules.

Keywords: drug resistance; cancer stem cells (CSCs); drug-likeness; pharmacokinetics; bioinformatics

\section{Introduction}

Cancer is one of the most prevalent and deadliest diseases globally, with an incident increase of approximately 14 million new cancer cases annually [1], and the total number of patients anticipated to be 450 million by 2025 [2]. There are several mechanisms associated with complexities in cancer, including survival strategies implemented by cancer cells to escape from cytotoxic therapies, which consequently lead to resistance to current therapeutic interventions [3]. In addition, immunotherapy has evolved as a new approach in oncology, and patients exhibit greater tolerance to this approach than to traditional alternative medicines [4]. However, cancers in most patients have been reported to be resistant to immuno-checkpoint inhibitors (ICIs) after a certain time [5]. Accumulating evidence has shown that another mechanism through which cancer develops and progresses results from changes in the cell cycle [6]. Recent studies have demonstrated that cyclin D1 (CCND1) plays a significant role in regulating the cell cycle, thereby promoting tumor proliferation, invasion, and metastasis [7], as well as angiogenesis and resistance to chemotherapy and radiotherapy in multiple cancer types [8,9]. Moreover, CCND1 overexpression has been reported in several cancers including lung cancer, breast cancer, colon cancer, glioblastomas, melanomas, and oral squamous cell carcinoma, with an amplification rate of approximately $40 \%$ [10]. This is associated with metastasis, negative responses to ICIs, and poor prognoses of solid tumors [11-13].

As an oncogene, $C C N D 1$ was shown to promote tumor growth by regulating cyclindependent kinase 4 (CDK4), through modulating the cell cycle transtion from the $\mathrm{G}_{1}$ to the $S$ phase, thereby making $C C N D 1$ and its regulatory partner, $C D K 4$, attractive potential therapeutic targets [14]. CCND1 treatment includes drugs that mainly target its transcription and protein synthesis; however, these current treatment options are still limited due to the resistance that eventually develops [15]. Therefore, novel therapeutic approaches are needed that give rise to better responses. Since $C D K 4$ is activated by $C C N D 1$, recent studies have shown that it is also overexpressed in several cancer types and shares similar oncogenic characteristic with CCND1 in tumor tissues [16,17]. Additionally, cell cycle progression is also regulated by one of the serine/threonine protein kinases: polo-like kinase 1 (PLK1) [18]. Recently, the amplification and overexpression of PLK1 were reported in a variety of tumors [19]. Its role in cancer includes cell differentiation and tumor progression, consequently resulting in poor clinical outcomes and therefore promoting the need for PLK1 inhibitors in cancer [20,21]. In addition, emerging studies have shown that the suppression of PLK1 by current inhibitors results in the downregulation of CCND1 overexpression in tumors [22,23]. Additionally, the overexpression of PLK1 in tumor cells was shown to activate cancer stem cells (CSCs), with the cluster of differentiation 44 (CD44) surface marker mostly being expressed [24].

Comprehensive computational software has been utilized most recently in drug target discovery, by applying in silico bioinformatics predictions for drug targets, as well as screening cancer cells at the gene level in a search for novel drug targets [25]. Moreover, the use of available online databases has improved data analysis and drug target identifica- 
tion [26]. NSC765600 and NSC765691 are both small molecules, respectively derived from diflunisal (Pubchem CID: 3059) and fostamatinib (Pubchem CID: 11671467) [27,28], which were chemically synthesized in our lab [29], where we produced open-ring (NSC765600) and closed-ring (NSC765691) derivatives. In this study, we applied drug target predictions and identified CCND1/CDK4/PLK1/CD44 as potential drug candidates of NSC765600 and NSC76569, and further performed molecular docking, which revealed putative binding energies of CCND1/CDK4/PLK1/CD44 with NSC765600 and NSC76569. Furthermore, we showed that both NSC765600 and NSC76569 displayed antiproliferative and cytotoxic activities in vitro against a panel of NCI-60 human cancer cell lines.

\section{Materials and Methods}

\subsection{Pharmacokinetic (PK), Drug-Likeness, and Medicinal Chemical Analyses}

To analyze the drug-likeness, medicinal chemistry, PKs, and the adsorption, distribution, metabolism, excretion, and toxicity (ADMET) properties of our compounds (NSC765600 and NSC765691), we utilized the SwissADME software developed by the Swiss Institute of Bioinformatics (http:/ / www.swissadme.ch, 27 February 2021) [30]. Druglikeness properties were analyzed according to the Lipinski (Pfizer) rule-of-five, Ghose (Amgen), Veber (GSK), Egan (Pharmacia), and Veber (GSK); in addition, the Lipinski (Pfizer) rule-of-five defines four simple physicochemical parameters with the following ranges (molecular weight $\leq 500, \log p \leq 5$, hydrogen bond donors $\leq 5$, and hydrogen bond acceptors $\leq 10$ ) [31] for drug-likeness and drug discovery. A bioavailability score was calculated based on the probability of the compound having at least $0.1(10 \%)$ oral bioavailability in rats or measurable Caco-2 permeability [32], while gastrointestinal absorption (GIA) and brain penetration properties were analyzed using the brain or intestinal estimated permeation (BOILED-Egg) model [33].

\subsection{Identifying the Molecular Targets of NSC765600 and NSC765691}

Target genes of NSC765600 and NSC765691 were predicted using the Swiss target prediction online tool (http:/ / www.swisstargetprediction.ch/, 27 February 2021), which uses the principle of similarity to predict the drug target. The predictions are also based on "probability", which is derived from the target score to assess the likelihood of the predicted targets being correct, and the values are based on the correspondence of the average precision (i.e., number of true-positives divided by the total number of predicted targets at different thresholds). These predictions were then used in in vitro and in vivo experiments [34]. Furthermore, we used the DTP-COMPARE algorithms [35] to identify the fingerprint (activity patterns) of NSC765600 and NSC765691, which was based on the correlation of the compounds with NCI synthetic compounds and standard agents. Herein, we used the NSC IDs of both compounds as "seed for the COMPARE algorithms".

\subsection{Bioinformatics Predictions}

The micro-RNA (mRNA) levels of differentially expressed genes (DEGs) of CCND1, CDK4, PLK1, and CD44 in tumorous versus normal tissues from various cancers in The Cancer Genome Atlas (TCGA) database were analyzed using UALCAN (http:/ / ualcan.path.uab.edu/, 28 March 2021) and GEPIA2 (http:/ /gepia2.cancer-pku.cn/\#index, 28 March 2021 bioinformatics software with default settings.

\subsection{Protein-Protein Interaction (PPI) Analysis}

In a further analysis, we applied the GeneMANIA (https:/ / genemania.org/, 22 February 2021) and STRING (https: / / string-db.org/, 22 February 2021) online analytical tools to predict PPIs between expressed genes, and a gene ontology (GO) enrichment analysis including biological processes and Kyoto Encyclopedia of Genes and Genomes (KEGG) pathways. The results displayed in a network for PPIs indicated correlations of CDK4 with CCND1, CDK4 with CD44, CCND1 with CD44, CDK4 with PLK1, and PLK1 with CCND1, wherein nodes symbolize genes and edges represents networks. 


\subsection{Analysis of Genomic Alterations of CCND1/CDK4/PLK1/CD44 in Multiple Cancer Types}

To analyze genomic alterations of $C C N D 1 / C D K 4 / P L K 1 / C D 44$ gene signatures, we applied the Oncoprint feature of cBioportal software (https://www.cbioportal.org/, 14 March 2021), which categorizes gene alterations based on percentages of individual genes due to amplification, and in a further analysis, we determined the alteration frequencies of our gene signatures in multiple cancer types. Next, we used a mutually exclusivity panel analysis, a sub-tool of cBioportal software, to determine if the remaining altered genes within the entire set co-occurred with CCND1/CDK4/PLK1/CD44 signatures, and the threshold was designed with $p<0.001$.

\subsection{In Silico Analysis of Molecular Docking of Receptors and Ligands}

The potential inhibitory activities of NSC765600 and NSC765691 were analyzed by the in silico molecular docking of oncogene signaling of CCND1, CDK4, PLK1, and CD44, compared to the standard inhibitors fascaplysin, ribociclib, and volasertib of CCND1, CDK4, and PLK1, respectively. The 3D structures of NSC765600 and NSC765691 were drawn in sybyl mol2 using the Avogadro molecular builder and visualization tool (http:/ / avogadro. openmolecules.net, 8 February 2021) [36]. The 3D structural conformers of fascaplysin (CID: 7329), ribociclib (CID: 4431912), and volasertib (CID: 10461508) were downloaded in SDF file format from the PubChem database. The mol2 and SNF format structures were subsequently converted to PDB format using the Pymol molecular visualization system (https:/ / pymol.org/2/, 8 February 2021), and later to the PDBQT file format using autodock tool software (http:/ / autodock.scripps.edu/resources/adt, 8 February 2021). Crystal structures of CCND1 (PDB-6P8G), CDK4 (PDB-4O9W), PLK1 (PDB-2W9F), and CD44 (PDB-1UUH) were retrieved from the Protein Data Bank (PDB) (https:/ / www.rcsb. org/, 8 February 2021), in PDB format, and eventually converted to PDBQT files with the autodock tool. Molecular docking was performed using autodock tools [37]. For visualization, we used pymol, and further interactive 3D visualization and interpretation were analyzed using the BIOVIA discovery studio tool [38].

2.7. In Vitro Screening of NSC765600 and NSC765691 against Full National Cancer Institute (NC I)-60 Cell Panels of Human Tumor Cell Lines

NSC765600 and NSC765691 were submitted for screening to the NCI (Rockville, MD 20850, USA) to screen its panel of NCI-60 cancer cell lines. The two compounds were screened for antiproliferative and cytotoxic effects at an initial single dose $(10 \mu \mathrm{M})$ against the full NCI-60 cell line panel, which includes leukemia, non-small cell lung cancer (NSCLC), melanomas, renal cancer, breast cancer, central nervous system (CNS) cancers, ovarian cancer, and prostate cancer, in agreement with the protocol of the NCI (http:/ / dtp.nci.nih.gov, 1 January 2021).

\subsection{Data Analysis}

Pearson's correlations were used to assess correlations between CCND1/CDK4/PLK1/ $C D 44$ expressions in multiple cancer types. The statistical significance of DEGs was evaluated using the Wilcoxon test. ${ }^{*} p<0.05$ was accepted as being statistically significant. The Kaplan-Meier curve was employed to present patient survival in different cancer cohorts.

\section{Results}

\subsection{NSC765600 and NSC765691 Adhere to the Required Drug-Likeness Criteria}

NSC765600 (open ring) (Figure 1a) [27,39] and NSC765691 (closed ring) (Figure 1b) are respective fostamatinib- and salicylanilide-derived compounds [40]. Using a computer simulation, we applied the criteria described for drug-likeness concepts to identify the potential of these novel compounds for drug discovery and development [41]. The GIA and blood-brain barrier (BBB) permeability of these small compounds were evaluated using the BOILED-Egg predictive model [33]. Both NSC765600 and NSC765691 exhibited prospective GIA and BBB permeability. Six important properties for oral bioavailability, 
which enabled us to assess the drug-likeness of NSC765600 and NSC765691, were evaluated as displayed on the bioavailability radar (Supplementary Figure S1). The compounds were evaluated according to their molecular weight, polarity, solubility, saturation, flexibility, and lipophilicity, and both met drug-likeness requirements. Interestingly, NSC765600 and NSC765691 showed favorable PKs, drug-likeness, and medicinal chemical properties, and met the Lipinski's rule-of-five for drug-likeness and drug discovery, with good synthetic accessibilities of 2.64 and 3.48, respectively (Tables 1 and 2). The bioavailability of the compounds based on GIA revealed a score of 0.55 (55\%) for both compounds, which indicates acceptable PK properties. Since both compounds met the drug-likeness criteria, we further investigated the activity patterns (fingerprints) and mechanistic relations of NSC765600 and NSC765691 with NCI synthetic compounds and standard anticancer agents, as stipulated by the development therapeutics program (DTP). This program is used to identify the molecular targets and mechanisms of an unknown compound from an available known compound in the NCI databases [42]. After a comparative analysis, as anticipated, NSC765600 and NSC765691 shared similarities fingerprints and mechanisms with NCI synthetic compounds and standard anticancer agents, with Pearson's correlations of $p=0.46 \sim 0.25$ for NSC765600 and $p=0.5 \sim 0.4$ for NSC76569 (Table 3).
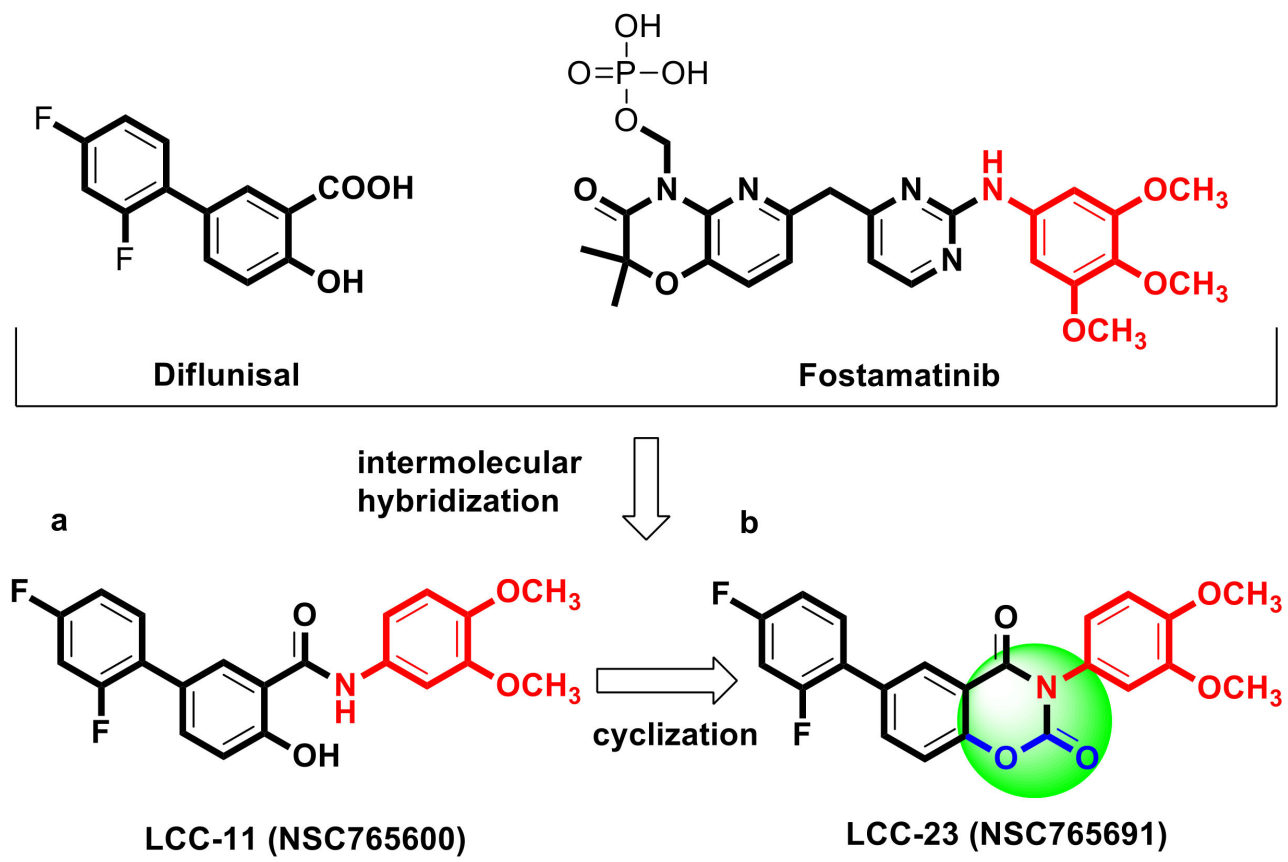

Figure 1. NSC765600 (a) and NSC765691 (b) are both small molecules derived from diflunisal (Pubchem CID: 3059) and fostamatinib (Pubchem CID: 11671467), respectively. 
Table 1. Physiochemical properties, pharmacokinetics, drug-likeness, and medical chemistry of NSC765600.

\begin{tabular}{|c|c|c|}
\hline \multicolumn{2}{|c|}{ Physicochemical Properties Based on Bioavailability Radar of NSC765600 } & Recommended Value \\
\hline Formula & \multicolumn{2}{|c|}{$\mathrm{C}_{21} \mathrm{H}_{17} \mathrm{~F}_{2} \mathrm{NO}_{4}$} \\
\hline Molecular weight & $385.36 \mathrm{~g} / \mathrm{mol}$ & $150-500 \mathrm{~g} / \mathrm{mol}$ \\
\hline Fraction Csp3 & 0.10 & $\leq 1$ \\
\hline Number of rotatable bonds & 6 & $\leq 10$ \\
\hline Number of H-bond acceptors & 6 & $\leq 12$ \\
\hline Number of H-bond donors & 2 & $\leq 5$ \\
\hline Molar refractivity & 101.02 & \\
\hline TPSA & $67.79 \AA^{2}$ & $\leq 140 \AA^{2}$ \\
\hline $\log P_{\mathrm{o} / \mathrm{w}}(\mathrm{XLOGP3})$ & 5 & $-0.7-5$ \\
\hline $\log S(E S O L)$ & -5.89 & $0-6$ \\
\hline \multicolumn{3}{|c|}{ Pharmacokinetics } \\
\hline GI absorption & High & \\
\hline $\mathrm{BBB}$ & Low & \\
\hline \multicolumn{3}{|c|}{ Drug-likeness } \\
\hline Lipinski & Yes; 0 violation & \\
\hline Ghose & Yes & \\
\hline Veber & Yes & \\
\hline Egan & Yes & \\
\hline Muegge & Yes & \\
\hline Bioavailability Score & $0.55(55 \%)$ & \\
\hline \multicolumn{3}{|c|}{ Medical Chemistry } \\
\hline Synthetic accessibility & 2.64 & $\begin{array}{l}1 \text { (easy to make) and } 10 \text { (difficult to } \\
\text { make) }\end{array}$ \\
\hline
\end{tabular}

Table 2. Physiochemical properties, pharmacokinetics, drug-likeness, and medical chemistry of NSC765691.

\begin{tabular}{|c|c|c|}
\hline \multicolumn{2}{|c|}{ Physicochemical Properties Based on Bioavailability Radar of NSC765691 } & Recommended Value \\
\hline Formula & \multicolumn{2}{|c|}{$\mathrm{C}_{22} \mathrm{H}_{15} \mathrm{~F}_{2} \mathrm{NO}_{5}$} \\
\hline Molecular weight & $411.36 \mathrm{~g} / \mathrm{mol}$ & $150-500 \mathrm{~g} / \mathrm{mol}$ \\
\hline Fraction Csp3 & 0.09 & $\leq 1$ \\
\hline Number of rotatable bonds & 4 & $\leq 10$ \\
\hline Number of H-bond acceptors & 7 & $\leq 12$ \\
\hline Number of H-bond donors & 0 & $\leq 5$ \\
\hline Molar refractivity & 106.42 & \\
\hline TPSA & $70.67 \AA^{2}$ & $\leq 140 \AA^{2}$ \\
\hline $\log \mathrm{P}_{\mathrm{o} / \mathrm{w}}(\mathrm{XLOGP3})$ & 4.53 & $-0.7-5$ \\
\hline \multirow[t]{2}{*}{$\log S(E S O L)$} & -5.52 & $0-6$ \\
\hline & \multicolumn{2}{|l|}{ Pharmacokinetics } \\
\hline GI absorption & High & \\
\hline \multirow[t]{2}{*}{ BBB } & Yes $(0.215)$ & \\
\hline & Drug-likeness & \\
\hline Lipinski & Yes; 0 violation & \\
\hline Ghose & Yes & \\
\hline Veber & Yes & \\
\hline Egan & Yes & \\
\hline Muegge & Yes & \\
\hline Bioavailability score & $0.55(55 \%)$ & \\
\hline \multicolumn{3}{|c|}{ Medical Chemistry } \\
\hline Synthetic accessibility & 3.48 & 1 (easy to make) and 10 (difficult to make) \\
\hline
\end{tabular}


Table 3. NCI synthetic compounds and standard anticancer agents sharing similar anticancer fingerprints and mechanistic correlations with NSC765600 and NSC765691.

\begin{tabular}{|c|c|c|c|c|c|c|c|c|c|}
\hline & Rank & $p$ & CCLC & Target Descriptor & MW & $p$ & CCLC & Target Descriptor & MW \\
\hline \multirow{11}{*}{ 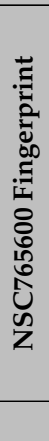 } & 1 & 0.63 & 49 & Dinoterb & 240.21 & 0.46 & 52 & 4-ipomeanol & 168.19 \\
\hline & 2 & 0.62 & 50 & 8-[(4-tert-butylphenoxy)] & 342.4 & 0.41 & 49 & Piperazine & 86.14 \\
\hline & 3 & 0.59 & 53 & Masterid & 360.5 & 0.4 & 44 & Amsacrine & 393.5 \\
\hline & 4 & 0.59 & 52 & Nitrodan(usan) & 296.3 & 0.4 & 52 & Fluorodopan & 249.67 \\
\hline & 5 & 0.58 & 41 & Resorcinol, 4-hexyl-(8ci) & 194.27 & 0.39 & 42 & Mustard & 159.08 \\
\hline & 6 & 0.58 & 52 & Azd- 4635 & 315.73 & 0.37 & 50 & Tamoxifen & 371.15 \\
\hline & 7 & 0.57 & 50 & Chimaphilin & 186.21 & 0.35 & 52 & Topotecan & 421.4 \\
\hline & 8 & 0.56 & 53 & 10074-g5 & 332.3 & 0.33 & 44 & Morpholino & 86.11 \\
\hline & 9 & 0.56 & 53 & Gsk586581a & 381.4 & 0.32 & 52 & Procarbazine & 221.3 \\
\hline & 10 & 0.55 & 50 & Tioconazole (usan) & 387.7 & 0.28 & 45 & Diaziquone & 364.35 \\
\hline & Rank & $\mathbf{r}$ & CCLC & Target Descriptor & MW & $\mathbf{r}$ & CCLC & Target Descriptor & MW \\
\hline \multirow{10}{*}{ 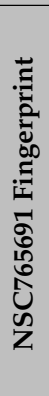 } & 1 & 0.69 & 41 & Flavanone & 298.3 & 0.5 & 56 & 4-ipomeanol & 168.19 \\
\hline & 2 & 0.69 & 54 & 13668 & 217.69 & 0.49 & 53 & Piperazine & 86.14 \\
\hline & 3 & 0.68 & 56 & Isomammein & 372.5 & 0.47 & 55 & Tamoxifen & 371.5 \\
\hline & 4 & 0.66 & 57 & C.I. 37525 & 311.8 & 0.46 & 56 & Flavoneacetic & 280.5 \\
\hline & 5 & 0.65 & 56 & $10074-g 5$ & 332.3 & 0.44 & 56 & Sulfoximine & 64.09 \\
\hline & 6 & 0.64 & 47 & 4-(acetyl) amphilectolide & 302.4 & 0.43 & 45 & Mustard & 159.08 \\
\hline & 7 & 0.64 & 53 & Chimaphilin & 186.21 & 0.42 & 55 & Bryostatin & 905 \\
\hline & 8 & 0.64 & 57 & Niclosamide (usan) & 327.12 & 0.41 & 56 & Glycoxalic acid & 74.03 \\
\hline & 9 & 0.63 & 46 & Thiazolobenzimidazole & 288.32 & 0.4 & 56 & Fluorodopan & 249.67 \\
\hline & 10 & 0.63 & 56 & Azd- 4635 & 315.73 & 0.4 & 56 & Merbarone & 263.27 \\
\hline
\end{tabular}

$p$-Pearson's correlation coefficient (value ranges between -1 and 1 (values becomes more significant as they increase above)). CCLCcommon cell lines count cell counts. MW—Molecular weight $(\mathrm{g} / \mathrm{mol})$.

\subsection{CCND1/CDK4/PLK1/CD44 Gene Signatures are Potential Drug Targets for NSC765600 and NSC765691}

We applied the Swiss target prediction tool to NSC765600 and NSC765691 and identified potential druggable genes, including MTOR, PIK3R1, GSK3A, PIK3CD, PLK1, CCND1, CDK2, and CDK4 for NSC765600 and PLK1, CCND1, TLR9, GSK3B, PTK2, and CDKs, among other target genes, for NSC765691. There are various target classes that the prediction tool identified for both compounds, such as kinases, enzymes, family A G protein-coupled receptors, proteases, phosphodiesterase, isomerase, oxidoreductase, and Toll-like and interleukin (IL)-1 receptors. The calculation results from the software were based on the "probability values" derived from the target score to assess the likelihood of the predictions being correct. Herein the probability showed a similar value of 0.11 across all predicted target genes. (Supplementary Figures S2 and S3, Tables 4 and 5). 
Table 4. Common names, Uniprot and ChEMBL IDs, and target classes of specific gene targets of NSC765600.

\begin{tabular}{|c|c|c|c|c|}
\hline Target & Common Name & Uniprot ID & ChEMBL ID & Target Class \\
\hline $\begin{array}{c}\text { Serine/threonine-protein } \\
\text { kinase MTOR }\end{array}$ & MTOR & P42345 & CHEMBL2842 & Kinase \\
\hline $\begin{array}{c}\text { PI3-kinase p85-alpha } \\
\text { subunit }\end{array}$ & PIK3R1 & P27986 & CHEMBL2506 & Enzyme \\
\hline $\begin{array}{l}\text { Serotonin } 2 \mathrm{c}(5-\mathrm{HT} 2 \mathrm{c}) \\
\text { receptor }\end{array}$ & HTR2C & P28335 & CHEMBL225 & $\begin{array}{l}\text { Family A G } \\
\text { protein-coupled } \\
\text { receptor }\end{array}$ \\
\hline Cyclin-dependent kinase 4 & $C D K 4$ & P11802 & CHEMBL331 & Kinase \\
\hline Matrix metalloproteinase 3 & MMP3 & P08254 & CHEMBL283 & Protease \\
\hline Histone chaperone ASF1A & ASF1A & Q9Y294 & CHEMBL3392950 & Unclassified protein \\
\hline $\begin{array}{c}\text { PI3-kinase p110-delta } \\
\text { subunit }\end{array}$ & PIK3CD & O00329 & CHEMBL3130 & Enzyme \\
\hline Phosphodiesterase 5A & PDE5A & O76074 & CHEMBL1827 & Phosphodiesterase \\
\hline CDK2/Cyclin A & CCNA2 CDK2 & P20248 P24941 & CHEMBL3038469 & Kinase \\
\hline Nuclear factor NF-kappa-B & NFKB1 & P19838 & CHEMBL3251 & Other cytosolic protein \\
\hline $\begin{array}{l}\text { Cyclin-dependent kinase } \\
\text { 2/cyclin E }\end{array}$ & CCNE2CDK2 CCNE1 & O96020 P24941 P24864 & CHEMBL2094126 & Other cytosolic protein \\
\hline $\begin{array}{c}\text { Serine/threonine-protein } \\
\text { kinase PLK1 }\end{array}$ & PLK1 & P53350 & CHEMBL3024 & Kinase \\
\hline Beta-glucuronidase & GUSB & P08236 & CHEMBL2728 & Enzyme \\
\hline $\begin{array}{c}\text { cAMP-dependent protein } \\
\text { kinase alpha-catalytic } \\
\text { subunit }\end{array}$ & PRKACA & P17612 & CHEMBL4101 & Kinase \\
\hline Interleukin-8 receptor B & CXCR2 & P25025 & CHEMBL2434 & $\begin{array}{l}\text { Family A G } \\
\text { protein-coupled } \\
\text { receptor }\end{array}$ \\
\hline $\begin{array}{c}\text { Dual specificity tyrosine- } \\
\text { phosphorylation-regulated } \\
\text { kinase 1B }\end{array}$ & DYRK1B & Q9Y463 & CHEMBL5543 & Kinase \\
\hline $\begin{array}{c}\text { Cyclin-dependent kinase } \\
\text { 4/cyclin D1 }\end{array}$ & CCND1 CDK4 & P24385 P11802 & CHEMBL1907601 & Kinase \\
\hline Cyclophilin A & PPIA & P62937 & CHEMBL1949 & Isomerase \\
\hline $\begin{array}{l}\text { Platelet-derived growth } \\
\text { factor receptor alpha }\end{array}$ & PDGFRA & P16234 & CHEMBL2007 & Kinase \\
\hline $\begin{array}{l}\text { Glycogen synthase } \\
\text { kinase-3 alpha }\end{array}$ & GSK3A & P49840 & CHEMBL2850 & Kinase \\
\hline
\end{tabular}


Table 5. Common names, Uniprot and ChEMBL IDs, and target classes of specific gene targets of NSC765691.

\begin{tabular}{|c|c|c|c|c|}
\hline Target & Common Name & Uniprot ID & ChEMBL ID & Target Class \\
\hline $\begin{array}{l}\text { Serine/threonine-protein } \\
\text { kinase }\end{array}$ & PLK1 & P53350 & CHEMBL3024 & Kinase \\
\hline $\begin{array}{c}\text { Nicotinamide } \\
\text { phosphoribosyl transferase }\end{array}$ & NAMPT & P43490 & CHEMBL1744525 & Enzyme \\
\hline $\begin{array}{l}\text { Rho-associated protein } \\
\text { kinase } 1\end{array}$ & ROCK1 & Q13464 & CHEMBL3231 & Kinase \\
\hline Monoamine oxidase B & MAOB & P27338 & CHEMBL2039 & Oxidoreductase \\
\hline Focal adhesion kinase 1 & PTK2 & Q05397 & CHEMBL2695 & Kinase \\
\hline $\begin{array}{l}\text { Vascular endothelial } \\
\text { growth factor receptor } 2\end{array}$ & KDR & P35968 & CHEMBL279 & Kinase \\
\hline $\begin{array}{c}\text { Tyrosine-protein kinase } \\
\text { TIE-2 }\end{array}$ & TEK & Q02763 & CHEMBL4128 & Kinase \\
\hline $\begin{array}{l}\text { Cyclin-dependent kinase } \\
5 / \text { CDK5 activator } 1\end{array}$ & CDK5R1 CDK5 & Q15078 Q00535 & CHEMBL1907600 & Kinase \\
\hline Cyclin-dependent kinase 7 & CDK7 & P50613 & CHEMBL3055 & Kinase \\
\hline $\begin{array}{l}\text { Platelet-derived growth } \\
\text { factor receptor alpha }\end{array}$ & PDGFRA & $\mathrm{P} 16234$ & CHEMBL2007 & Kinase \\
\hline TGF-beta receptor type I & TGFBR1 & P36897 & CHEMBL4439 & Kinase \\
\hline Phosphodiesterase 5A & PDE5A & O76074 & CHEMBL1827 & Phosphodiesterase \\
\hline $\begin{array}{c}\text { Cyclin-dependent kinase } \\
\text { 4/cyclin D1 }\end{array}$ & CCND1 CDK4 & P24385 P11802 & CHEMBL1907601 & Kinase \\
\hline $\begin{array}{c}\text { Rho-associated protein } \\
\text { kinase } 2\end{array}$ & ROCK2 & O75116 & CHEMBL2973 & Kinase \\
\hline Cyclin-dependent kinase 2 & CDK2 & P24941 & CHEMBL301 & Kinase \\
\hline Cyclin-dependent kinase 1 & CDK1 & P06493 & CHEMBL308 & Kinase \\
\hline Cyclin-dependent kinase 4 & $C D K 4$ & P11802 & CHEMBL331 & Kinase \\
\hline $\begin{array}{c}\text { G-protein coupled receptor } \\
\text { kinase } 2\end{array}$ & GRK2 & P25098 & CHEMBL4079 & Kinase \\
\hline $\begin{array}{l}\text { Toll-like receptor } \\
\text { (TLR7/TLR9) }\end{array}$ & TLR9 & Q9NR96 & CHEMBL5804 & $\begin{array}{l}\text { Toll-like and Il-1 } \\
\text { receptors }\end{array}$ \\
\hline $\begin{array}{l}\text { Glycogen synthase } \\
\text { kinase- } 3 \text { beta }\end{array}$ & GSK3B & P49841 & CHEMBL262 & Kinase \\
\hline
\end{tabular}

\subsection{CCND1/CDK4/PLK1/CD44 are Overexpressed in Multiple Cancers and Associated with Poor Prognoses}

An analysis using the UALCAN (http:/ / ualcan.path.uab.edu/, 28 March 2021) online bioinformatics tool with default settings showed that the mRNA levels of CCND1/CDK4/ PLK1/CD44 were higher in tumor tissues, compared to normal tissues, in multiple cancer types (Figure 2). To further investigate the correlations between CCND1/CDK4/PLK1/CD44 siganature expressions and clinical prognoses, we predicted the overall survival percentage using multiple datasets, which is a sub-tool of survival analysis on the GEPIA2 software (http://gepia2.cancer-pku.cn/, 28 March 2021). Based on our analysis, we found that CCND1/CDK4/PLK1/CD44 signatures were linked with shorter survival percentages in a group of multiple cancer types, including bladder carcinoma, breast cancer, colon adenocarcinoma, glioblastoma multiforme, head and neck cancers, leukemia, NSCLC, and ovarian cancer (Figure 3). 
A

Expression of CCND1 across TCGA cancers (with tumor and normal samples)

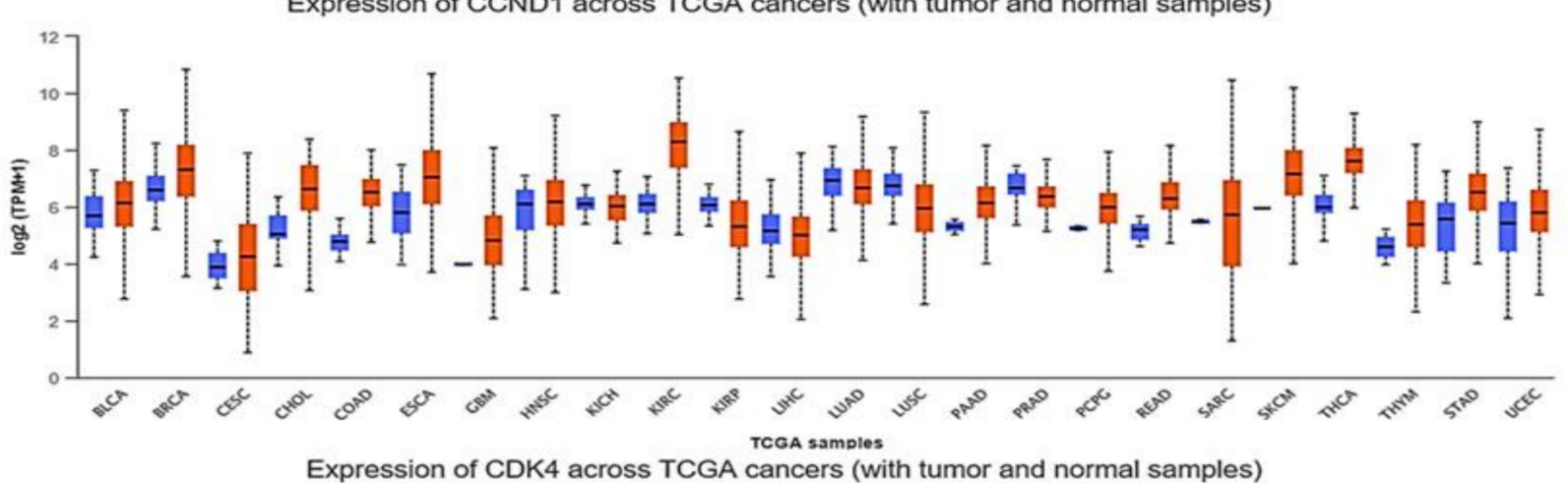

B

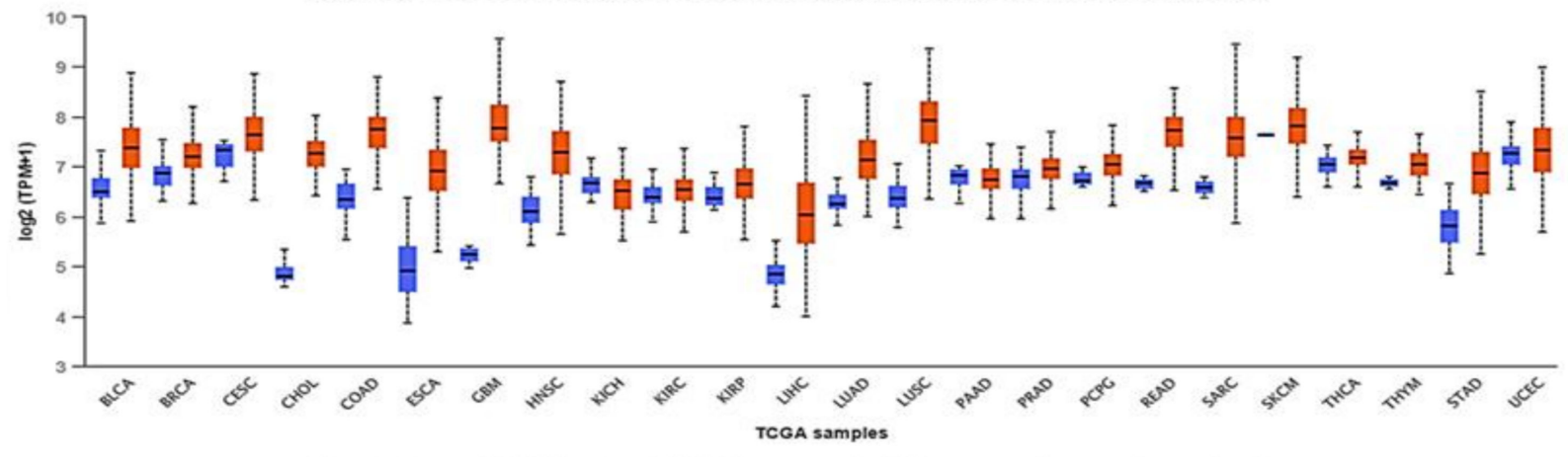

C

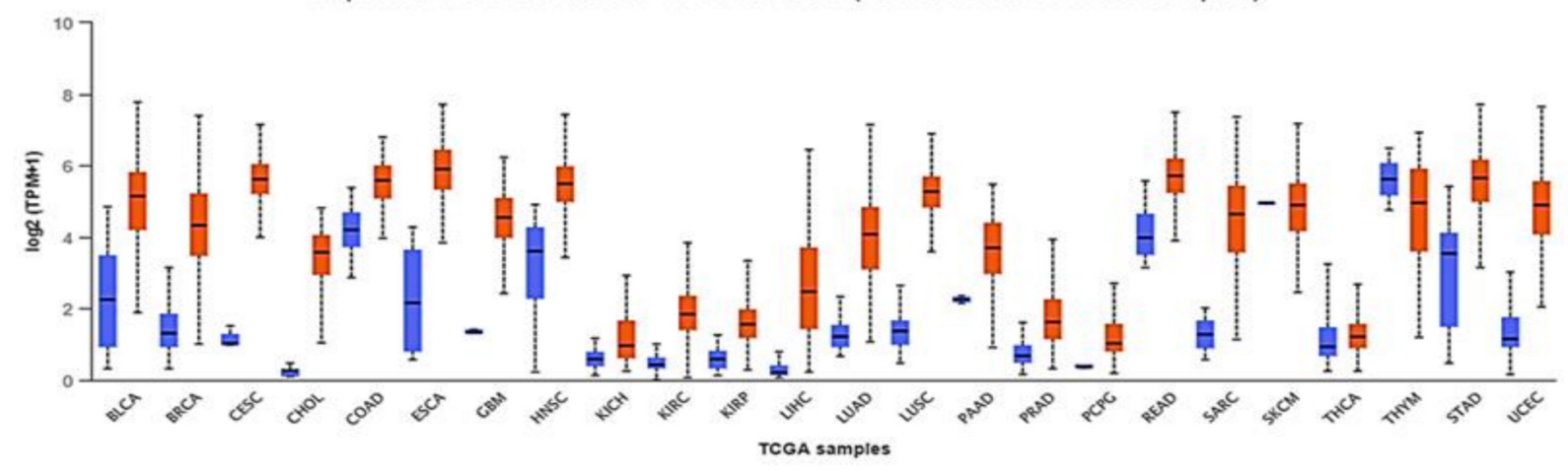

D

Expression of CD44 across TCGA cancers (with tumor and normal samples)

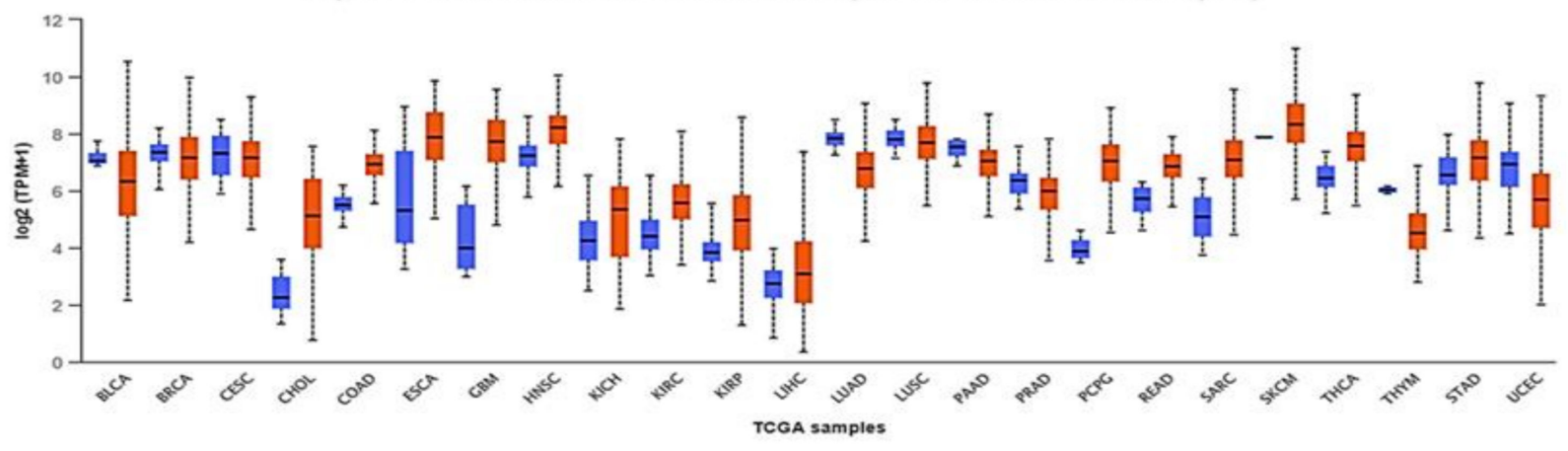

Figure 2. Cyclin D1 (CCND1)/cyclin-dependent kinase 4 (CDK4)/polo-like kinase 1 (PLK1)/cluster of differentiation 44 (CD44) genes are highly expressed in multiple cancer types. TCGA dataset of differential expression levels of (A) CCND1, (B) CDK4, (C) PLK1, and (D) CD44, based on patients with tumor tissues (red) and normal tissues (blue). 

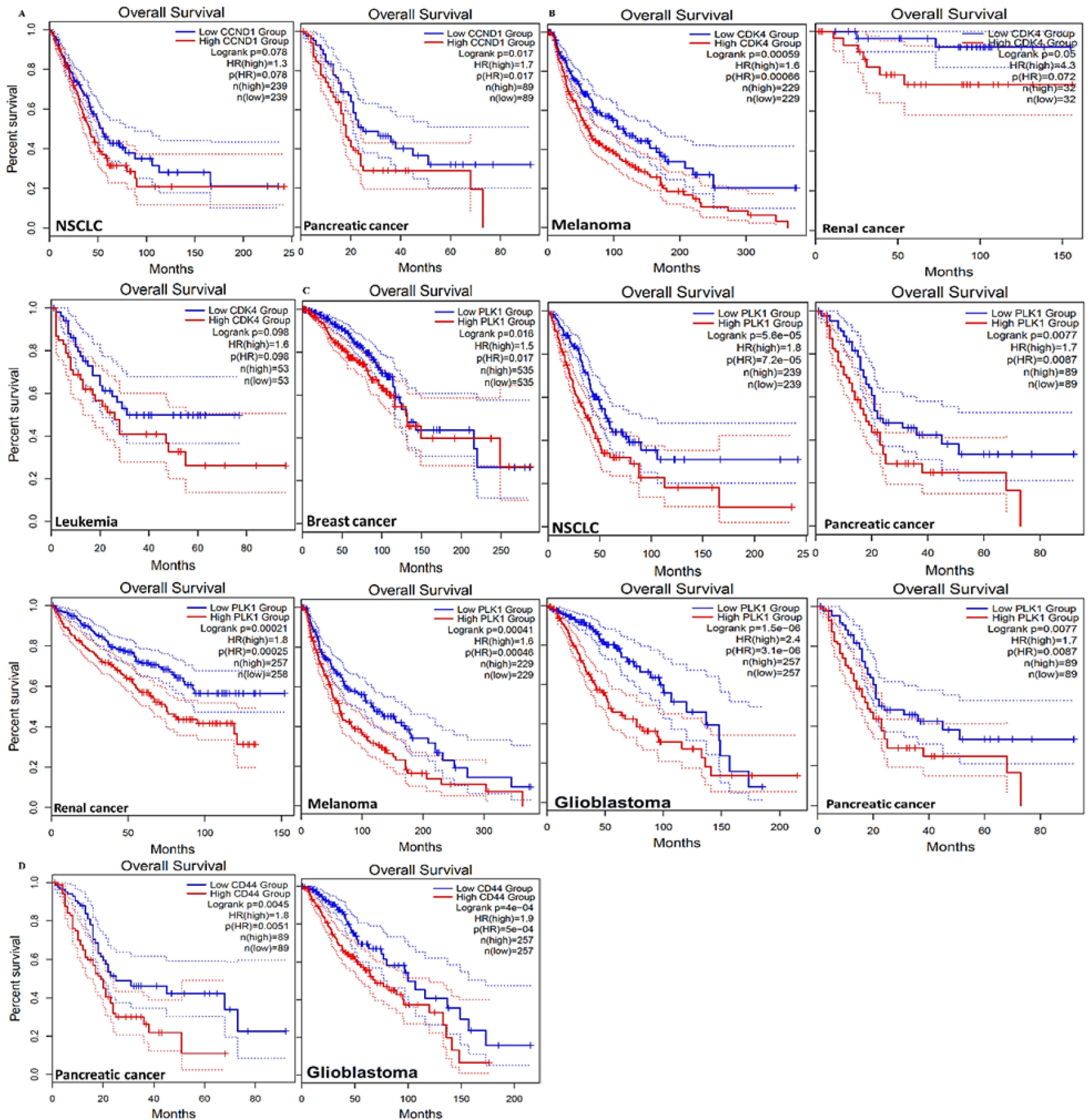

Figure 3. Predictions of overall survival percentages using CCND1/CDK4/PLK1/CD44. Increased mRNA levels of CCND1, CDK4, PLK1 and CD44 oncogenic signaling were found to be associated with shorter survival percentages in the following selected cancer types: NSCLC, pancreatic cancer, melanoma, renal cancer, leukemia, breast cancer and glioblastoma multiforme. The mRNA levels of (A) CCND1 (B) CDK4 (C) PLK1 and (D) CD44 were shown to be associated with short survival percentages in the abovementioned cancer types, with hazard ratio $>1$ and $p<0.05$ considered to be significant.

Moreover, we explored the GEPIA tool and Human Protein Atlas (HPA) database for immunohistochemistry (IHC) (HPA; www.proteinatlas.org/, 27 March 2021), to compare the expression levels of CCND1, PLK1, and CD44 between tumor samples and normal samples. HPA is a public database that displays IHC human protein expressions for tumor tissue and normal tissues [43]. Based on our analysis results, CCND1, PLK1, and CD44 genes' signaling were significantly expressed in multiple cancer types, including melanoma (SKMC), lung cancer (LAUD), ovarian cancer (OV), renal cancer (READ), bladder cancer 
(BLCA), colorectal cancer (COAD), prostate cancer (PRAD) and breast cancer (BRCA). In addition, the HPA data of CCND1, PLK1, and CD44 expression in tumor smaples exhibited high staining intensities, and all the IHC images were obtained from the HPA database to validate the expression of genes in the cancer types at the protein level. Staining intensity was analyzed based on low staining, medium staining, and high staining, and $p<0.05$ was considered statistically significant (Figure 4).

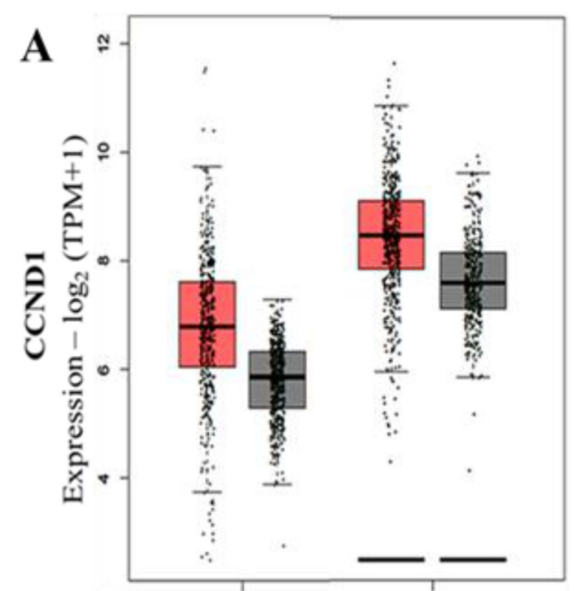

$(\operatorname{num}(\mathrm{T})=461 \operatorname{mum}(\mathrm{N})=558)(\operatorname{num}(\mathrm{T})=483 \operatorname{mum}(\mathrm{N})=347)$

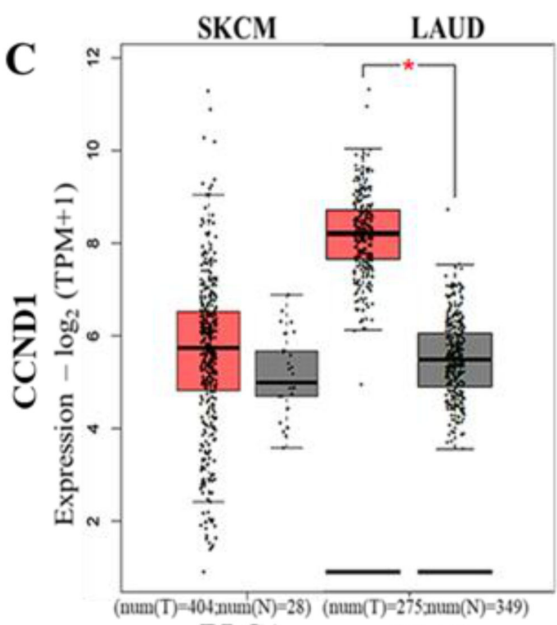

BLCA

COAD
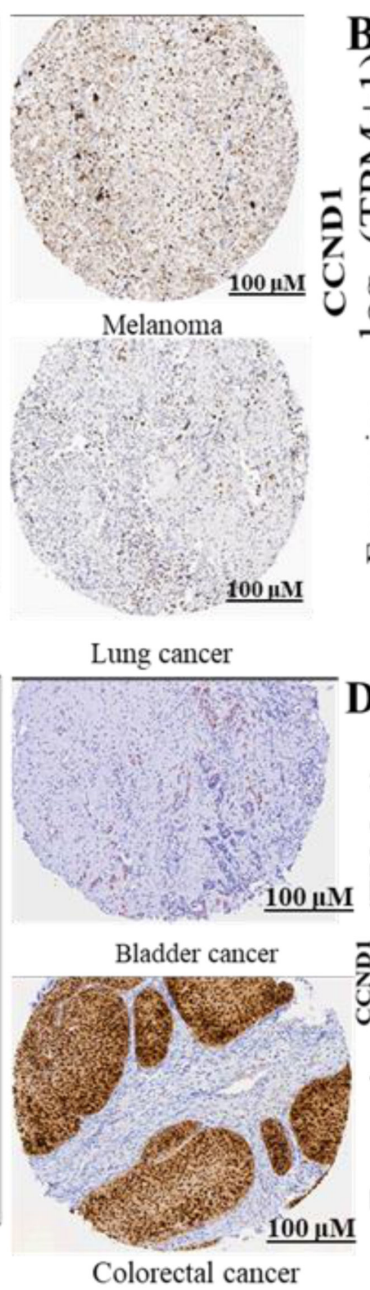

Colorectal cancer

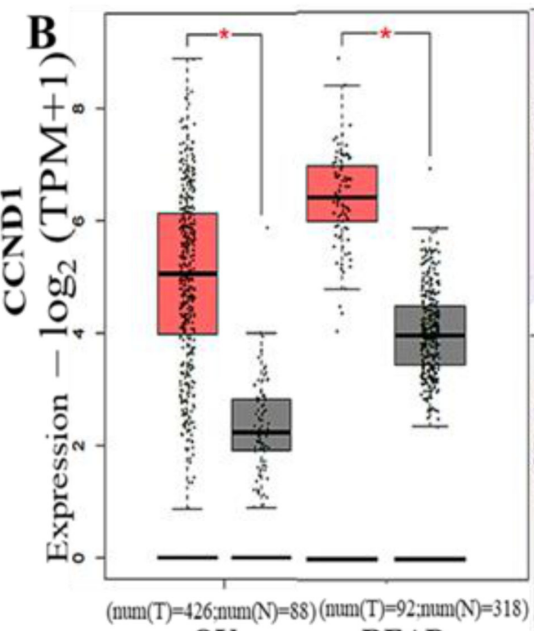

READ

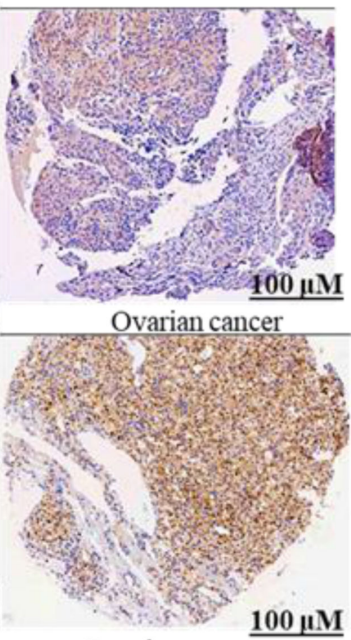

Renal cancer

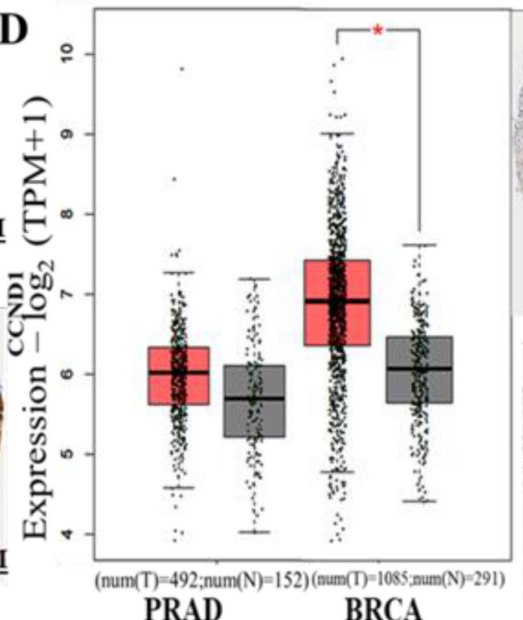

PRAD

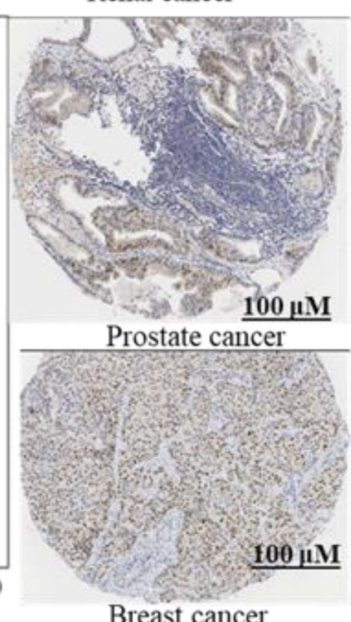

Breast cancer

Figure 4. Cont. 

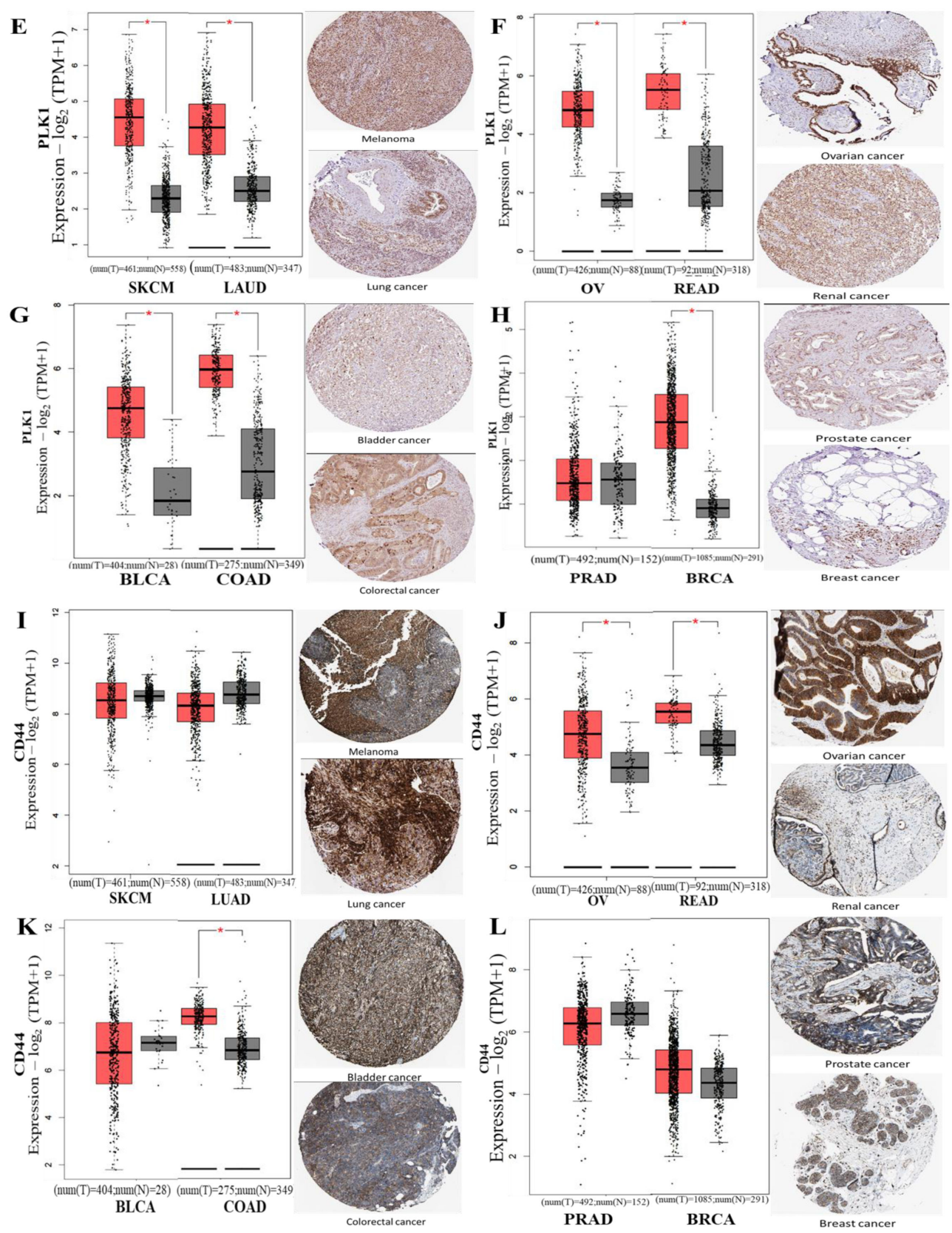

Figure 4. Validation of the expression of CCND1, CDK4 and PLK1 gene signtures on protein level using GEPIA and HPA databases. (A-D) CCND1, (E-H) PLK1, (I-L) CD44 GEPIA analysis expressed genes. HPA staining intensity was analyzed based on low staining, medium staining, and high staining. (A-E) CCND1 dispalyed low staining intensities in ovarian cancer and colorectal cancer, medium staining on breast cancer and prostate cancer, and high staining intensities on lung cancer, melanoma, renal cancer and bladder cancer. (E-H) PLK1 exhibited medium staining intensities on colorectal cancer, lung cancer, renal cancer, balddder cancer and prostate cancer, while high staining was observed on melanoma, ovarian cancer and breast cancera. (I-L) CD44 showed high staining intensities on all the abovementioned cancer types. Color images are available online. CDK4 was not included since the data have not yet been made available on the HPA database. The staining quality was $<75 \%$, and ${ }^{*} p<0.05$ was considered statistically significant, All images can be found online.

For futher analysis, we applied the GeneMANIA (https:/ / genemania.org/, 22 February 2021) and STRING databases (https:/ / string-db.org/cgi/, 22 February 2021) to predict PPIs between all four oncogenes. After considering the gene neighborhood, gene fusion, gene co-occurence, and coexpression, as anticipated, interactions were detected between CDK4 with CCND1, CDK4 with CD44, CDK4 with PLK1, and CCND1 with PLK1 within 
network clustering. The numbers of nodes and edges were ultimately increased to 24 and 192, respectively, within the network, with an interation enrichment average local clustering coefficient of 0.845 and $p<1.0 \times 10^{-16}$ (Figure 5A,B The accompanying table shows all other interacting proteins with CCND1/CDK4/PLK1/CD44, and the confidence cutoff value representing the interaction links was adjusted to 0.900 as the highest scoring link. In addition, we performed a gene enrichment analysis and predicted the major biological processes associated with the CCND1/CDK4/PLK1/CD44 gene signature. The correlated gene clusters were cellular protein modification processes, regeneration, DNA damage checkpoints, and transition of the mitotic cell cycle (Figure 5C). Moreover, CCND1/CDK4/PLK1/CD44 oncogenic signaling-correlated gene clusters affected 10 pathways, which were significantly associated with serveral functions, as shown in the KEGG pathways (Figure 5D. Pathways in cancer, the $P I 3 K-A K T$ signaling pathway, and prostate cancer were the most significant pathways associated with CCND1/CDK4/PLK1/CD44 siganling. Additionally, the seven other involved pathways included gliomas, NSCLC, gastric cancer, herpesviruses, hepatocellar carcinoma, breast cancer, and bladder cancer.

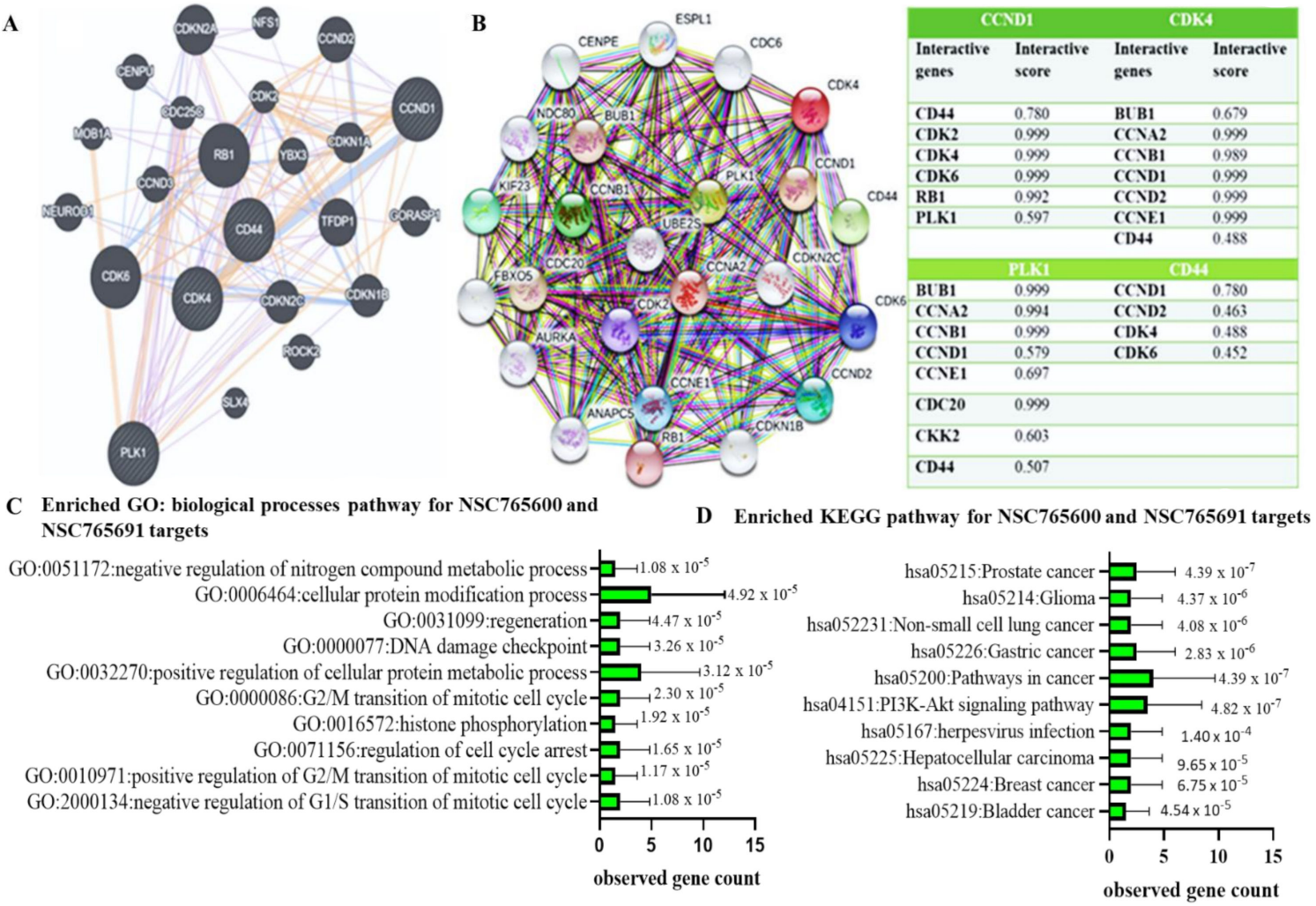

Figure 5. Protein-protein interaction (PPI) network among CCND1/CDK4/PLK1/CD44 genes in the GeneMANIA and STRING datasets. Interactions are shown after considering the gene neighborhood, gene fusion, gene co-occurance, and coexpression of CDK4 with CCND1, CDK4 with CD44,CDK4 with PLK1, and CCND1 with PLK1. (A) PPI network in the GeneMANIA dataset. (B) PPI network in the STRING dataset with network clustering. The numbers of nodes and edges were ultimately increased to 24 and 192, respectively, within the network, with an interation enrichment average local clustering coefficient of 0.845 and $p<1.0 \times 10^{-16}$. The accompanying table shows all other interacting proteins with CCND1/CDK4/PLK1/CD44, and the confidence cutoff value representing interaction links was adjusted to 0.900 as the highest scoring link. (C) Major biological processes associated with CCND1/CDK4/PLK1/CD44 oncogenic signature-correlated gene clusters. (D) KEGG pathway showing 10 affected pathways with CCND1/CDK4/PLK1/CD44 signatures, which were significantly associated with several functions. $p$-values are indicated in each panel. 


\subsection{CCND1/CDK4/PLK1/CD44 Genes are Altered in Multiple Cancer Types}

We applied the Oncoprint feature of cBioportal software (https:/ / www.cbioportal. org/, 14 March 2021), which categorized gene alterations of CCND1/CDK4/PLK1/CD44 based on percentages of separate genes due to amplification. The results of the analysis were as follows: $6 \%$ for CCND1, $2.8 \%$ for CDK4, 1.7\% for PLK1, and $1.6 \%$ for CD44 in multiple cancers, which included missense mutations (green), amplifications (red), deep deletions (blue), and no alterations (gray) (Figure 6A). In a further analysis, we determined the alteration frequencies of CCND1/CDK4/PLK1/CD44 gene signatures in multiple cancer types, and we then used a mutually exclusivity panel analysis, which is a sub-tool of the cBioportal software, and found the rest of the altered genes within the entire set that co-occurred with CCND1/CDK4/PLK1/CD44 signatures, at a threshold with $p<0.001$ as significant (Figure $6 \mathrm{~B}-\mathrm{E}$, and accompanying table).

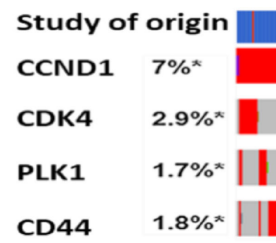

Acute Myeloid Leukemia (TCGA, PanCancer Atlas) | Bladder Urothelial Carcinoma (TCGA, PanCancer Atlas)

【Brain Lower Grade Glioma (TCGA, PanCancer Atlas) 【Breast Invasive Carcinoma (TCGA, PanCancer Atlas)

Colorectal Adenocarcinoma (TCGA, PanCancer Atlas) 【Glioblastoma Multiforme (TCGA, PanCancer Atlas)

I Head and Neck Squamous Cell Carcinoma (TCGA, PanCancer Atlas) Kidney Renal Clear Cell Carcinoma (TCGA, PanCancer Atlas) | Kidney Renal Papillary Cell Carcinoma (TCGA, PanCancer Atlas) 【 Lung Adenocarcinoma (TCGA, PanCancer Atlas)

B

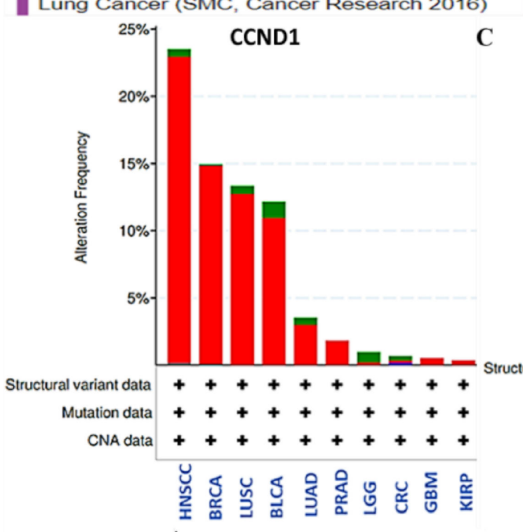

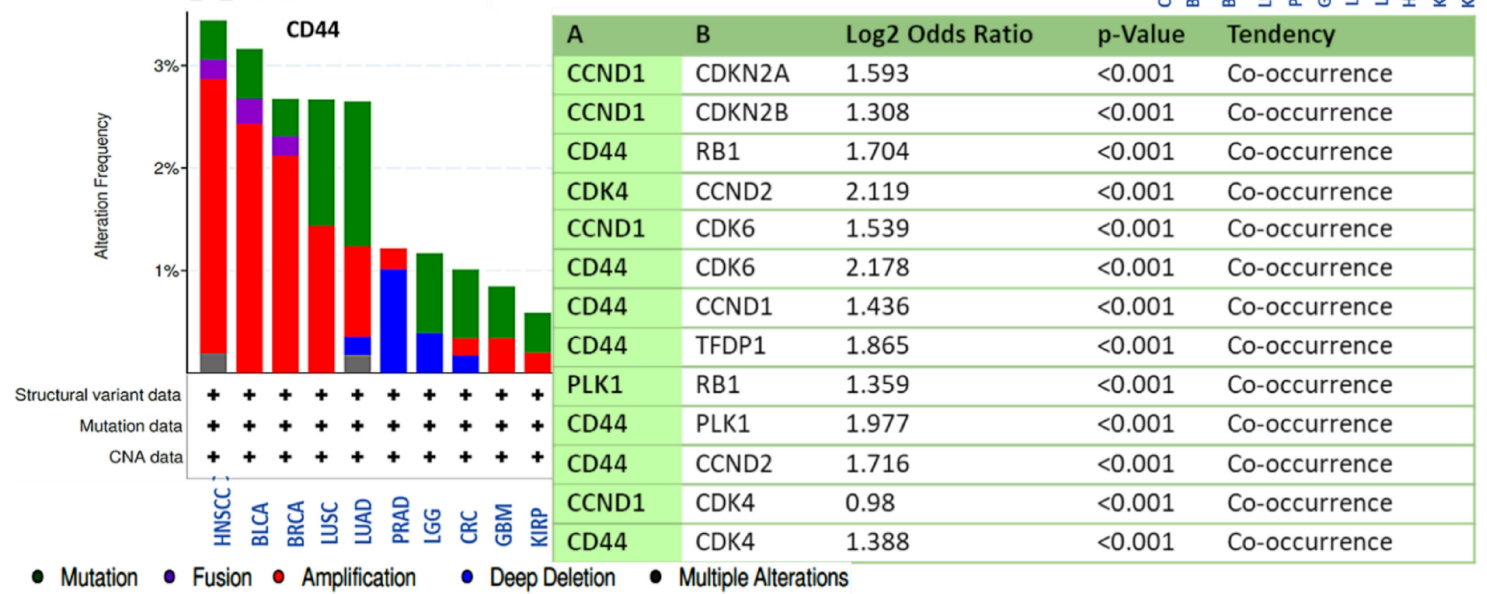

Figure 6. $C C N D 1 / C D K 4 / P L K 1 / C D 44$ oncogenes were amplified and found to co-occur in multiple cancer types. (A) Oncoprint analysis showed amplification (marked with *) of CCND1/CDK4/PLK1/CD44 based on percentages of separate genes, with $7 \%$ for CCND1, $2.9 \%$ for $C D K 4,1.7 \%$ for $P L K 1$, and $1.8 \%$ for $C D 44$ in multiple cancers, including missense mutations (green), amplifications (red), deep deletions (blue), or no alterations (gray). (B-E) Alteration frequencies of CCND1/CDK4/PLK1/CD44 signaling pathways in multiple cancer types. Accompanying table: co-occurrence of CCND1/CDK4/PLK1/CD44 signatures with other genes within the set, at a threshold of $p<0.001$ as significant. 


\subsection{Determining Protein-Ligand Interactions (PLIs)}

We applied molecular docking simulations, which demonstrated distinct binding abilities of NSC765600 and NSC765691 with CCND1/CDK4/PLK1/CD44 oncogenic signaling pathways. The two compounds achieved good binding affinities with the target proteins. From our docking analysis, the estimated binding energies $(\triangle \mathrm{G})$ of NSC765600 with CCND1 (PDB:6P8G), CDK4 (PDB:4O9W), PLK1 (PDB:2W9F), and CD44 (PDB-1UUH) complexes were $-9.3,-8.0,-7.4$, and $-7.0 \mathrm{kcal} / \mathrm{mol}$, respectively (Figure 7 , and accompanying table). Interestingly, NSC765691 displayed even greater binding free energies of $-9.6,-8.0,-7.7$, and $-7.3 \mathrm{kcal} / \mathrm{mol}$ with CCND1, CDK4, PLK1, and CD44, respectively (Figure 8 , and accompanying table). The docking analysis results have been visualized using Discovery studio, and the analysis has revealed the interactions of five conventional hydrogen bonds and their minimal distance constraints, including ASN198 (2.51 $)$ and ASN198 (2.04 ̊) with CCND1, ARG87 (2.29 A) with CDK4, SER439 (2.70 A) with PLK1, and CRY77 (2.90 ^) with CD44, all in complex with NSC765600.
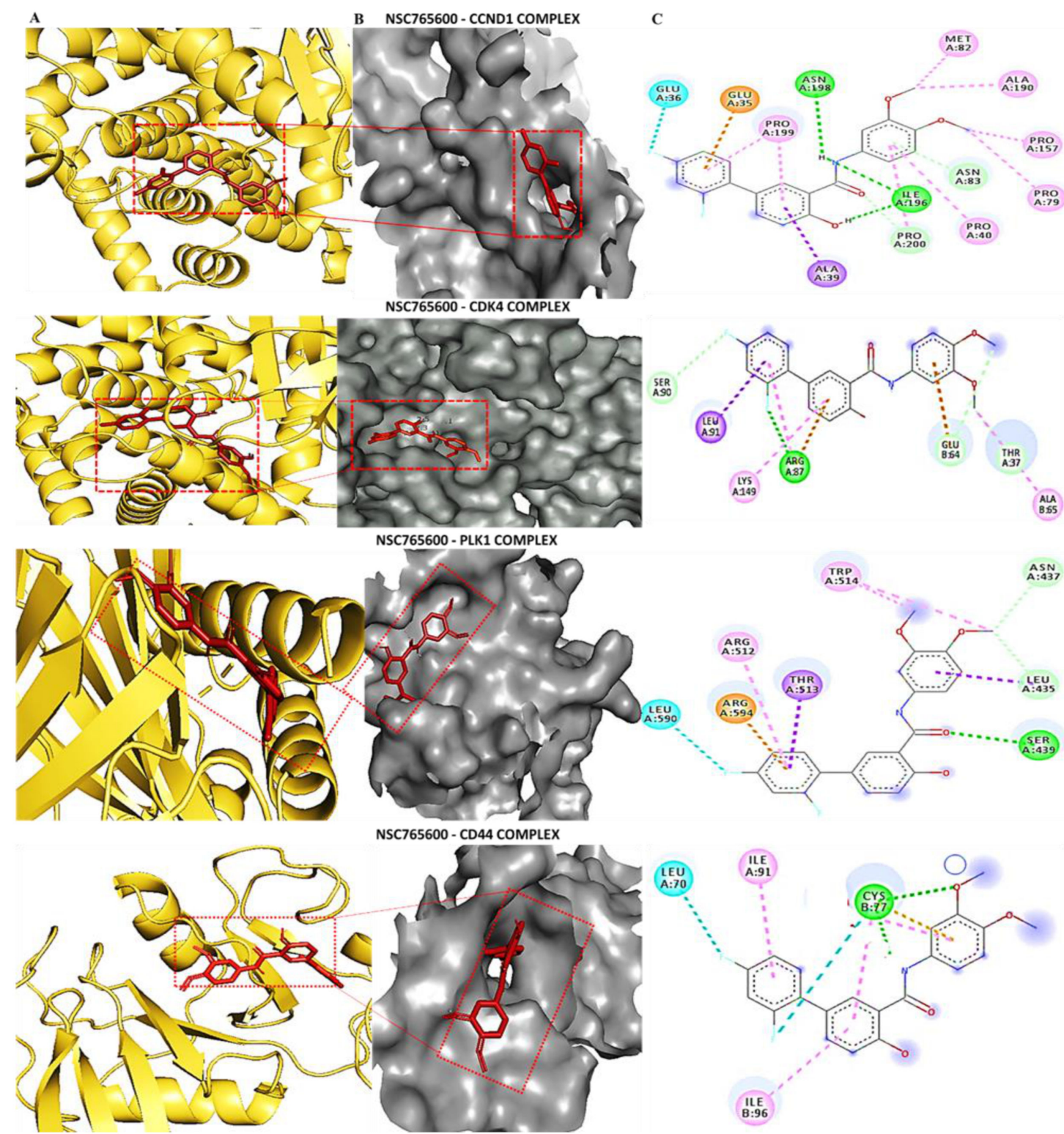

Figure 7. Cont. 


\begin{tabular}{|c|c|c|c|}
\hline \multicolumn{2}{|c|}{ NSC765600-CCND1 Complex $(\Delta \mathrm{G}=-9.3 \mathrm{Kcal} / \mathrm{mol})$} & \multicolumn{2}{|c|}{ NSC765600-CDK4 Complex $(\Delta G=-8.0 \mathrm{Kcal} / \mathrm{mol})$} \\
\hline $\begin{array}{l}\text { Type of interactions } \\
\text { and number of bonds }\end{array}$ & $\begin{array}{l}\text { Distance of interacting } \\
\text { Amino acids }\end{array}$ & $\begin{array}{l}\text { Type of interactions } \\
\text { and number of bonds }\end{array}$ & $\begin{array}{l}\text { Distance of interacting } \\
\text { Amino acids }\end{array}$ \\
\hline Conventional Hydrogen bond (2) & $\begin{array}{l}\text { ASN198 }(2.51 \AA) \text {, } \\
\text { ILE196 }(2.04 \AA),\end{array}$ & $\begin{array}{l}\text { Conventional Hydrogen } \\
\text { bond (1) }\end{array}$ & 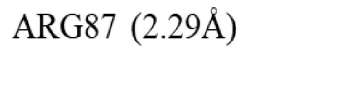 \\
\hline Carbon hydrogen bond (1) & PRO200 & Carbon hydrogen bond (2) & GLU64, \\
\hline Pi Anion (1) & GLU35 & & THR37 \\
\hline Pi-Sigma (1) & ALA39 & $\begin{array}{l}\text { Pi Anion } \\
\text { Pi-Sigma (1) }\end{array}$ & LEU91 \\
\hline Pi- Alkyl (6) & $\begin{array}{l}\text { MET82, ALA190, } \\
\text { PRO157, PRO79 } \\
\text { PRO40 } \\
\text { PRO199 }\end{array}$ & Pi- Alkyl (2) & $\begin{array}{l}\text { LYS149, } \\
\text { ALA65 }\end{array}$ \\
\hline \multicolumn{2}{|c|}{ NSC765600-PLK1 Complex $(\Delta G=-7.4 \mathrm{Kcal} / \mathrm{mol})$} & \multicolumn{2}{|c|}{ NSC765600-CD44 Complex $(\Delta G=-7.0 \mathrm{Kcal} / \mathrm{mol})$} \\
\hline $\begin{array}{l}\text { Type of interactions } \\
\text { and number of bonds }\end{array}$ & $\begin{array}{l}\text { Distance of interacting } \\
\text { Amino acids }\end{array}$ & $\begin{array}{l}\text { Type of interactions } \\
\text { and number of bonds }\end{array}$ & $\begin{array}{l}\text { Distance of interacting } \\
\text { Amino acids }\end{array}$ \\
\hline Conventional Hydrogen bond (1) & $\operatorname{SER} 439(2.70 \AA)$ & $\begin{array}{l}\text { Conventional Hydrogen } \\
\text { bond (1) }\end{array}$ & CYS77 $(2.90 \AA)$ \\
\hline Carbon hydrogen bond (2) & $\begin{array}{l}\text { ASN437, } \\
\text { LEU4354 }\end{array}$ & Carbon hydrogen bond & \\
\hline $\begin{array}{l}\text { Pi Anion (1) } \\
\text { Pi-Sigma (1) }\end{array}$ & $\begin{array}{l}\text { ARG594 } \\
\text { THR513 }\end{array}$ & $\begin{array}{l}\text { Pi Anion } \\
\text { Pi-Sigma }\end{array}$ & \\
\hline $\begin{array}{l}\text { Pi-Sigma (1) } \\
\text { Pi- Alkyl (2) }\end{array}$ & $\begin{array}{l}\text { THR513 } \\
\text { TRP514, } \\
\text { ARG512 }\end{array}$ & Pi- Alkyl (2) & $\begin{array}{l}\text { ILE91 } \\
\text { ILE96 }\end{array}$ \\
\hline
\end{tabular}

Figure 7. Docking profiles of NSC765600 with CCND1, CDK4, PLK1, and CD44. (A) Ligand-receptor interactions between CCND1/CDK4/PLK1/CD44 and NSC765600 in a two-dimensional representation. (B) Binding pocket presentation of the NSC7656000-CCND1/CDK4/PLK1/CD44 complex. (C) Visualization of putative docking poses of ligand-receptor interactions displayed by conventional hydrogen bonds. The accompanying table gives the binding energies of ligand-receptor interactions, including different types of interactions and the amino acid residues involved. 

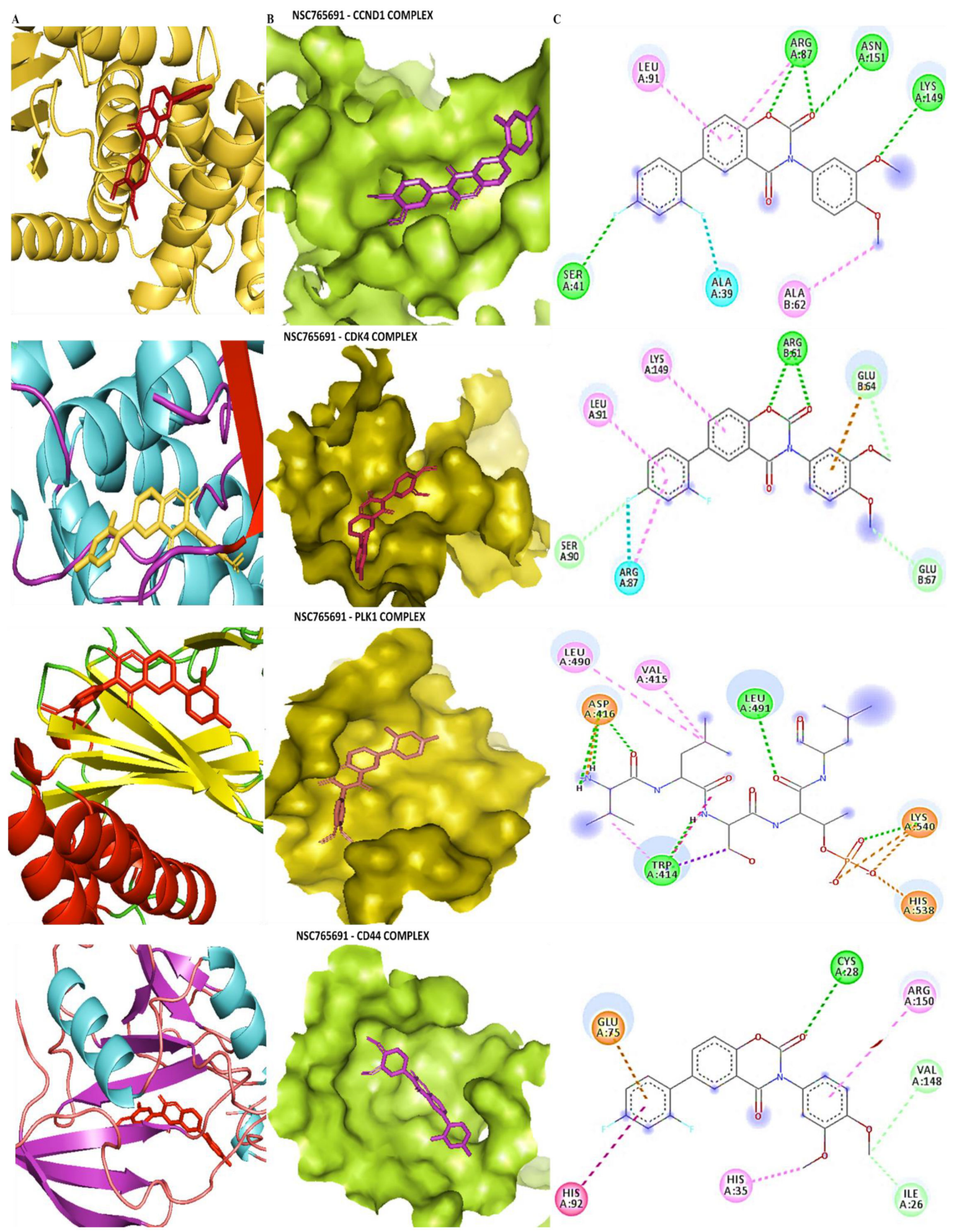

Figure 8. Cont. 


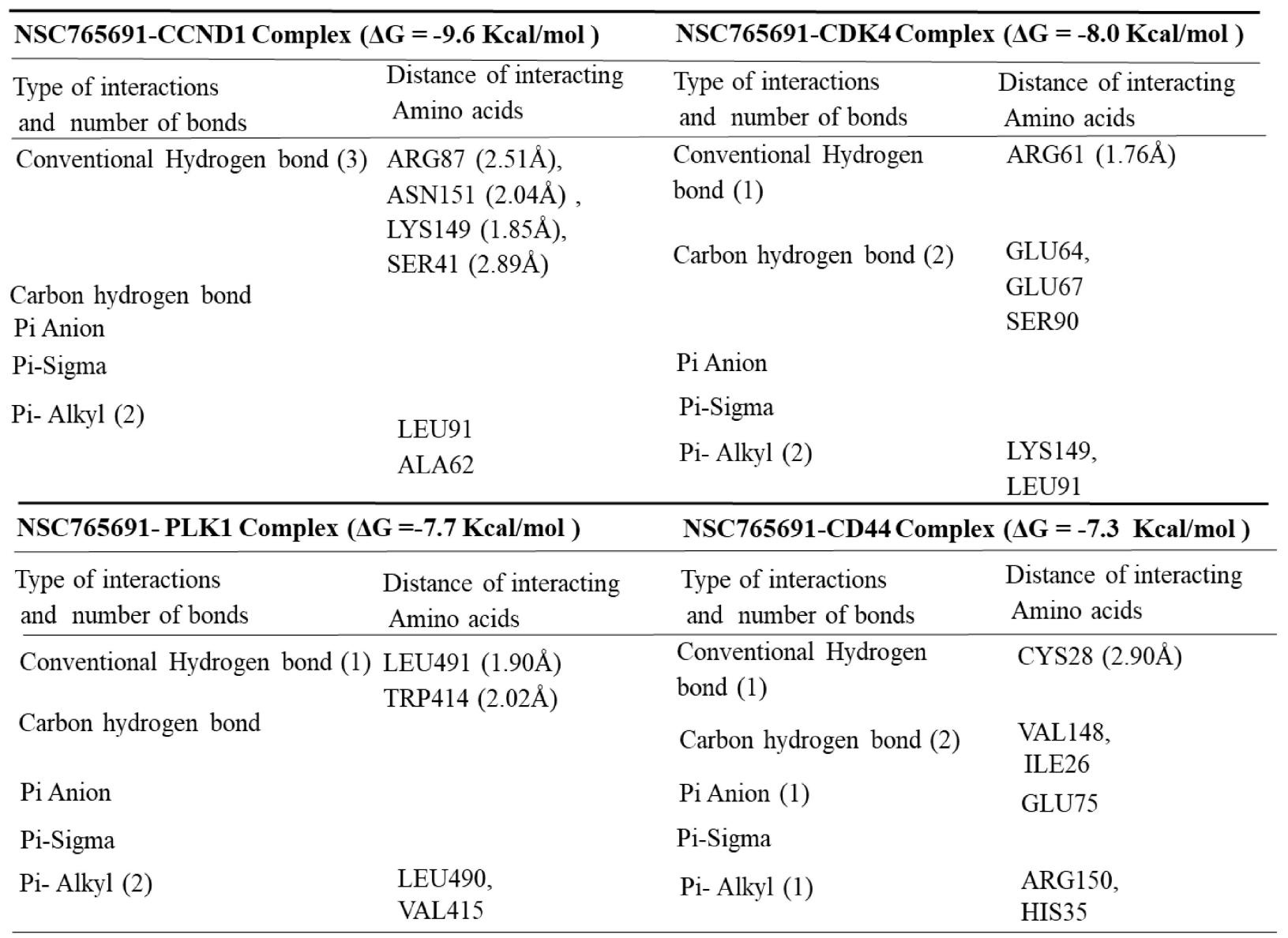

Figure 8. Docking profiles of NSC765691 with CCND1, CDK4, PLK1, and CD44. (A) Ligand-receptor interactions between CCND1/CDK4/PLK1/CD44 and NSC765691 in a two-dimensional representation. (B) Binding pocket presentation of the NSC7656000-CCND1/CDK4/PLK1/CD44 complex. (C) Visualization of putative docking poses of ligand-receptor interactions displayed by conventional hydrogen bonds. The accompanying table gives binding energies of ligand-receptor interactions, including different types of interactions and the amino acid residues involved.

Further supporting interactions were revealed, with their designated amino acids, including carbon-hydrogen bonds (PRO200), Pi-anion (GLU35), Pi-sigma (ALA39), and Pi-alkyl (MET82, ALA190, PRO157, PRO40, and PRO199) for the CCND1-NSC765600 complex; carbon-hydrogen bonds (GLU64, THR37), Pi-sigma (LEU91), and Pi-alkyl (LYS149 and ALA65) for the CDK4-NSC765600 complex; carbon-hydrogen bonds (ASN437 and LEU435), Pi-anion (ARG594), Pi-sigma (THR513), and Pi-alkyl (TRP514 and ARG512) for the PLK1-NSC765600 complex; and Pi-alkyl (ILE91 and ILE96) for the CD44-NSC765600 complex (Figure 7, and accompanying table). Moreover, the visualization analysis showed even more interactions of eight conventional hydrogen bonds and their minimal distance constraints, including ARG89 (2.51 $)$, ASN151 (2.04 $)$ ), LYS149 (1.85 $)$ ), and SER (2.89 $)$ with CCND1; ARG61 (1.76 ̊) with CDK4; LEU491 (1.90 ̊) and TRP414 (2.02 ̊) with PLK1; and CYS28 (2.09 A) with CD44, all in complex with NSC76591. Interactions were also further stabilized by other interactions with their assigned amino acids, including Pi-alkyl (LEU490 and VAL415) for the CCND1-NSC765691 complex; carbon-hydrogen bonds (GLU64, GLU67, and SER90), and Pi-alkyl (LYS149 and LEU91) for the CDK4NSC765691 complex; Pi-alkyl (LEU490 and VAL415) for the PLK1-NSC765691 complex; and carbon-hydrogen bonds (VAL148, ILE26, and GLU75) and Pi-alkyl (ARG150 and HIS35) for the CD44-NSC765600 complex (Figures 7 and 8, and accompanying tables). For further analysis, we compared the docking analysis results of NSC765600 and NSC765691 with standard inhibitors of fascaplysin, ribociclib, and volasertib for CCND1, CDK4, and 
PLK1, respectively. Interestingly, the standard inhibitors displayed the lowest binding free energies of -7.5 and $-7.6 \mathrm{kcal} / \mathrm{mol}$ for fascaplysin and ribociclib, respectively, but with the exception of volasertib, which showed a higher binding energy of $7.9 \mathrm{kcal} / \mathrm{mol}$, compared to our compounds (Figure 9). Therefore, the simulations predicted NSC765600 and NSC765691 to be potential multi-target inhibitors with high confidence.

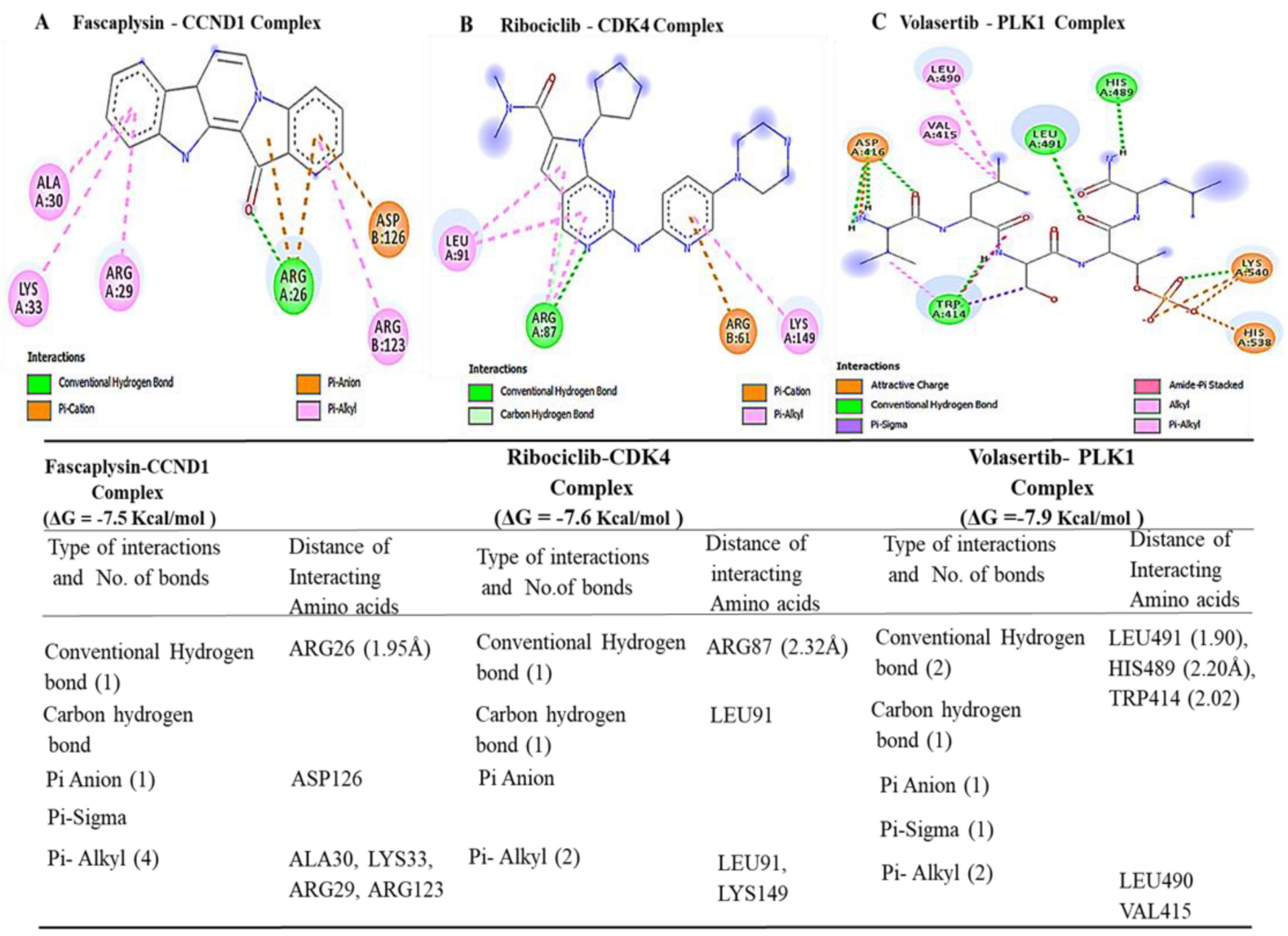

Figure 9. Docking profiles of the standard inhibitors fascaplysin, ribociclib, and volasertib with CCND1, CDK4, and PLK1, respectively. Visualization of the highest docking poses of ligand-receptor interactions displayed by conventional hydrogen bonds for the fascaplysin-CCND1 complex (A), ribociclib-CDK4 complex (B), and volasertib-PLK1 complex (C). the standard inhibitors displayed the lowest binding free energies of -7.5 and $-7.6 \mathrm{kcal} / \mathrm{mol}$ for fascaplysin and ribociclib, respectively, but with the exception of volasertib, which showed a higher binding energy of $7.9 \mathrm{kcal} / \mathrm{mol}$, compared to our compounds. The accompanying table gives the binding energies of ligand-receptor interactions, including different types of interactions and the amino acid residues involved.

\subsection{NSC765600 and NSC765691 Display Antiproliferative and Cytotoxic Effects against a Panel} of 60 Human Tumor Cell Lines

The anticancer activities of NSC765600 and NSC765691 were evaluated against a panel of 60 human cancer cell lines available from the US NCI-developed therapeutic program. This analysis included the antiproliferative and cytotoxic activities of the compounds on melanomas, central nevous system (CNS) cancers, renal cancer, breast cancer, NSCLC, leukemia, colon cancer, prostate cancer, and ovarian cancer [35]. The analytical results revealed the antiproliferative and cytotoxic effects of NSC765600 and NSC765691 against all cancer cell lines present in NCI data. The compunds exhibited anticancer activities after an initial dose of $10 \mu \mathrm{M}$, and the treatment efficacy was represented by the percentage (\%) growth (Figure 10). 


\section{NSC765600}

A
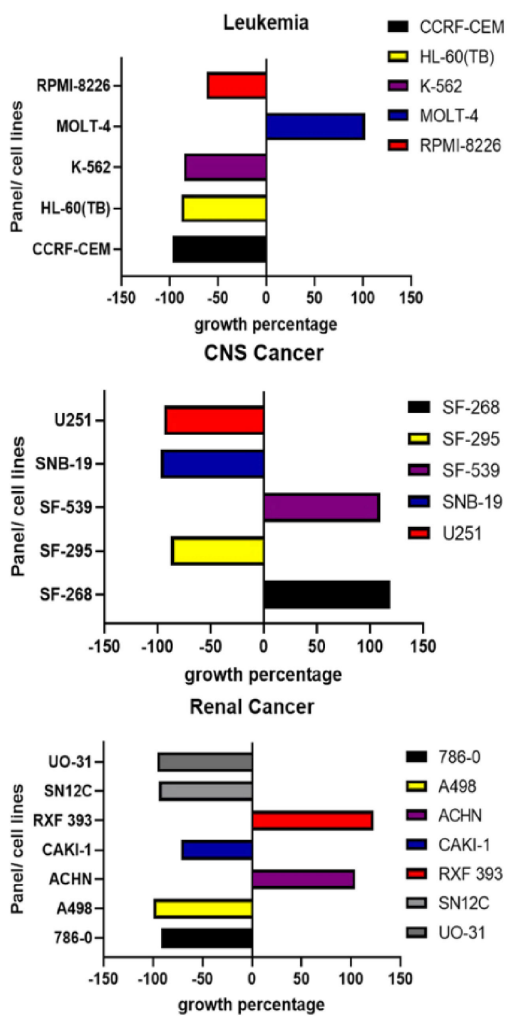
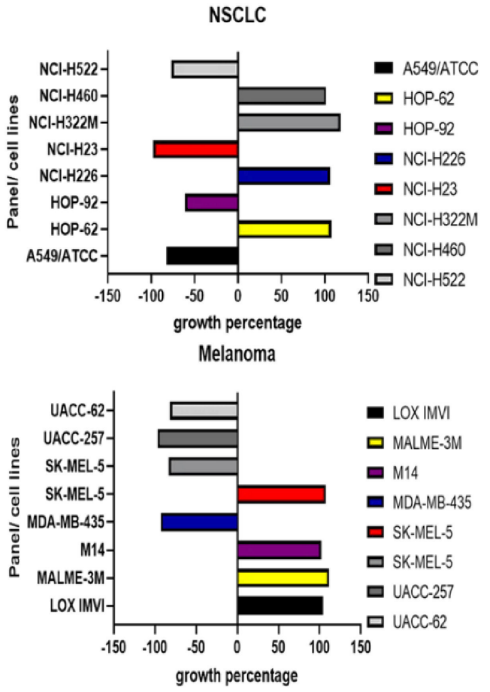

Prostate Cancer

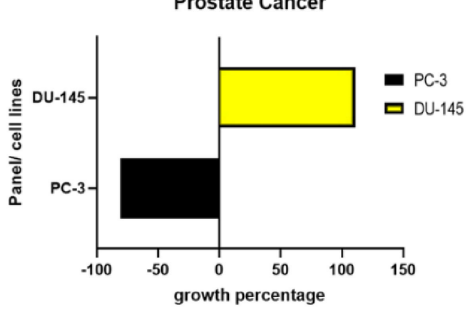

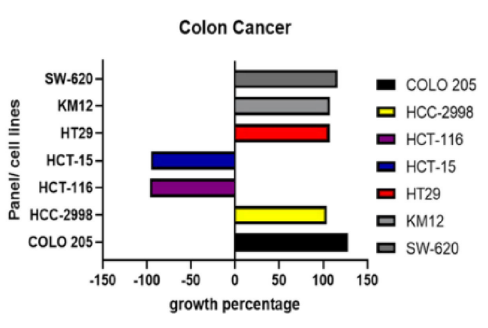

Ovarian Cancer
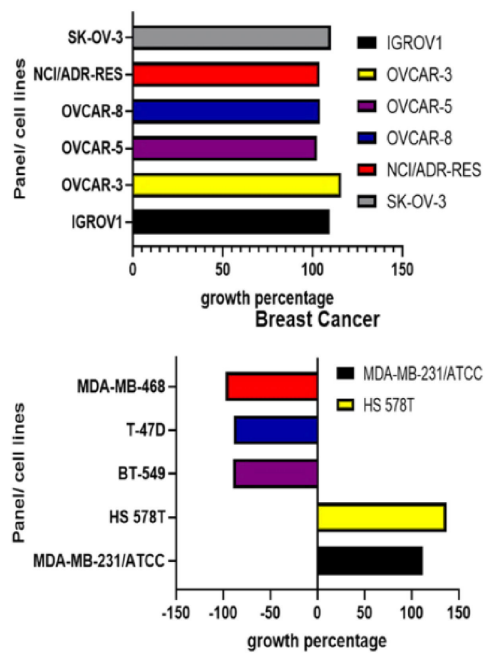

B
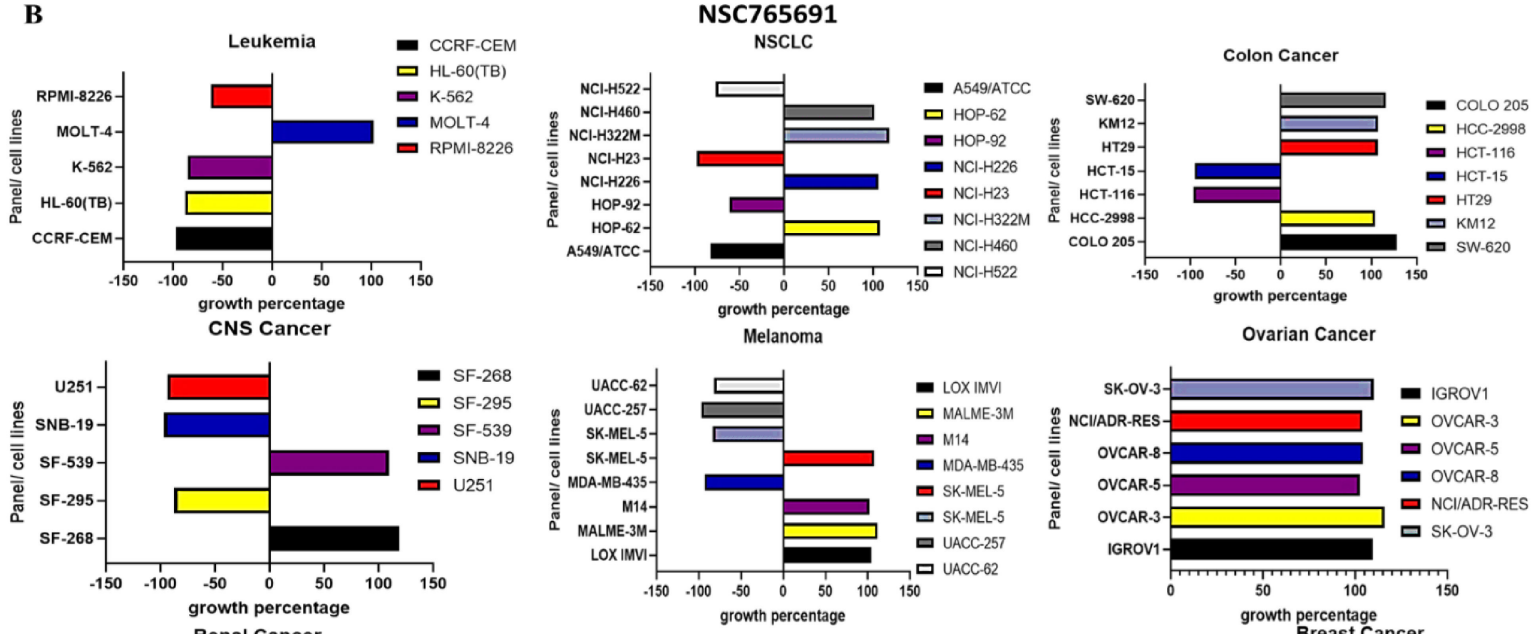

Ovarian Cancer
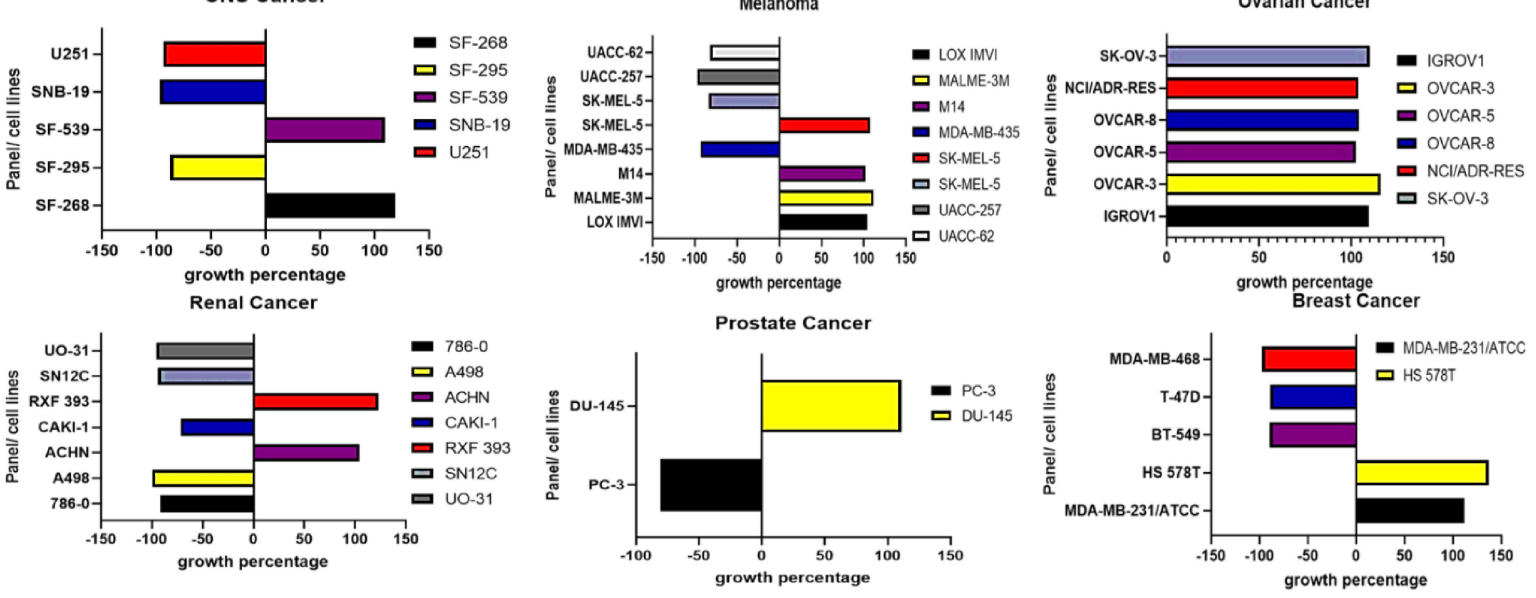

Figure 10. NSC765600 and NSC765691 exhibit anticancer activities against a panel of NCI-60 cancer cell lines. The sensitivities of the NCI-60 cancer cell lines were evaluated using (A) NSC765600 and (B) NSC765691 at an initial dose of $10 \mu \mathrm{M}$. The zero on the $x$-axis indicates the mean percentage of treated tumor cell lines. The anticancer effects of the compounds on the cell lines are represented by growth percentage (left) relative to mean growth percentage (right). When the growth percentage values are below zero (0), this indicates cell cytotoxicity/cell death, while when the mean growth percentage values are above 0 , this indicates antiproliferative effects. 


\section{Discussion}

Cancer progression has evolved over the years, and most fatalities now occur due to metastasis and resistance to therapeutic interventions [44]. Despite current advanced treatment interventions, including radiation, chemotherapy, and surgery, the median overall survival in patients with advanced disease is still under 5 years in the majority of cancers [5]. This therefore indicates the urgent need to develop novel and improved therapeutics that can be used either in combination with chemotherapy or as single agents. Recently, a vast amount of attention has been focused on small molecules as targeted treatments for cancer [42]. In this study, we revealed the inhibitory activities of two novel compounds, NSC765600 and NSC765691, in multiple cancers. Precepts of druglikeness allowed us to identify druggable targets in the initial stage of drug discovery and development [45]. Therefore, we utilized the SwissADME software developed by the Swiss Institute of Bioinformatics [30]; interestingly, the GIA and BBB permeability of these two small compounds exhibited good prospects. In addition, the compounds passed druglikeness requirements, and were evaluated according to their molecular weight, polarity, solubility, saturation, flexibility, and lipophilicity. Moreover, NSC765600 and NSC765691 showed favorable PK, drug-likeness, and medicinal chemical properties, and met Lipinski's rule-of-five for drug-likeness and drug discovery, with good synthetic accessibilities of 2.64 and 3.48, respectively (Tables 1 and 2). The bioavailability of the compounds based on GIA indicated a score of 0.55 (55\%) for both compounds, which indicates acceptable PK properties, implying that both NSC765600 and NSC765691 are drug-like compounds.

Since both compounds met the drug-likeness criteria, we further investigated the fingerprints and mechanistic relations of NSC765600 and NSC765691 with NCI synthetic compounds and standard anticancer agents, as stipulated by the DTP [42]. After a comparative analysis, as anticipated, NSC765600 and NSC765691 shared similar fingerprints and mechanisms with NCI synthetic compounds and standard anticancer agents, with Pearson's correlations of $p=0.46 \sim 0.25$ and $p=0.4 \sim 0.5$ for NSC765600 and NSC76569, respectively (Table 3 ). To further evaluate the biological and inhibitory effects of NSC765600 and NSC765691 in cancer, we identified CCND1/CDK4/PLK1/CD44 as potential druggable candidates for both compounds by using online prediction tools. Various target classes were also identified, among which were kinases and enzymes, suggesting that NSC765600 and NSC765691 are satisfactory compounds to be used as targets for CCND1/CDK4/PLK1/CD44 oncogenic signatures. The predictions were also based on "probability value", which is derived from the target score to assess the likelihood of the predicted targets being correct. The values were based on the correspondence of the average precision (i.e., number of true-positives divided by the total number of predicted targets at different thresholds) [34]. Herein, the results showed similar probability values of 0.11 across all target genes predicted to be correct. These predictions will be further used in in vitro and in vivo experiments.

In silico molecular docking is a computer simulation tool, which has been applied in drug design and development over the years, with the aim of predicting ligand-protein binding interactions [46]. Herein, we applied docking simulations of NSC765600 and NSC765691 with CCND1/CDK4/PLK1/CD44 oncogenic signaling pathways, and the results showed the unique interactions of NSC765600 and NSC765691 with the CCND1/CDK4/ PLK1/CD44 complex, and also exhibited predominant binding free energies for both compounds. Next, we compared the binding affinities of the NSC765600 and NSC765691 standard inhibitors, fascaplysin, ribociclib, and volasertib, with CCND1, CDK4, and PLK1, respectively. From the analysis, NSC765600 and NSC765691-CCND1, CDK4, PLK1, and CD44 complexes displayed the highest binding energies of $-9.3,-8.3,-7.4$ and $-7.0 \mathrm{kcal} / \mathrm{mol}$ and $(-9.6,-8.0,-7.7$ and $-7.3 \mathrm{kcal} / \mathrm{mol}$, respectively (Figures 7 and 8 and accompanying tables), as compared to the standard inhibitors' results, which were $-7.5,-7.6$ and $-7.9 \mathrm{kcal} / \mathrm{mol}$ for fascaplysin, ribociclib, and volasertib-CCND1, CDK4, and PLK1, respectively (Figure 9, accompanying table). Interestingly, NSC765691 (closed ring) showed even stronger binding affinities with the CCND1/CDK4/PLK1/CD44 oncogenes, compared to NSC765600 and selected standard inhibitors of CCND1/CDK4/PLK1. This confirms the 
findings from previous studies, which showed that closed-ring structures are more stable in drug design and development, compared to open-ring structures [47].

When we further applied a bioinformatics analysis using UALCAN and GEPIA2, we identified increased mRNA expression levels of CCND1/CDK4/PLK1/CD44 in multiple cancer types, which resulted in shorter overall survival times and poor prognoses in cancer patients, with p-values less than 0.05 and hazard ratios more than 1 considered to be significant (Figures 2 and 3). Additionally, PPIs showed interactions according to the gene neighborhood, gene fusion, gene co-occurance, and coexpression of $C D K 4$ with CCND1, CDK4 with CD44, CDK4 with PLK1, and CCND1 with PLK1, with an enrichment average local clustering coefficient of 0.845 and $p<1.0 \times 10^{-16}$. GO enrichment revealed major biological processes and pathways associated with CCND1/CDK4/PLK1/CD44 in different cancers (Figure 5). We further applied the cBioportal software and found that CCND1/CDK4/PLK1/CD44 genes were altered and co-occurring with other different genes in multiple cancers. The analysis showed the amplification of CCND1/CDK4/PLK1/CD44 based on percentages of separate genes, with $7 \%$ for CCND1, 2.9\% for CDK4, 1.7\% for PLK1, and $1.8 \%$ for $C D 44$ in multiple cancers, including the missense mutation amplifications, deep deletions, and alteration frequencies of these oncogenes.

Based on these finding, NSC765600 and NSC765691 exhibited potential inhibitory effects on the CCND1/CDK4/PLK1/CD44 oncogenic pathway, and passed all the required criteria for drug-likeness precepts as novel compounds. Further in vitro and in vivo studies in tumor-bearing mice will be performed to investigate the potential treatment efficacies of the novel NSC765600 and NSC765691 small molecules.

\section{Conclusions}

In conclusion, we have shown that CCND1/CDK4/PLK1/CD44 are potential drug targets for NSC765600 and NSC765691 small molecules, and the docking analytical results revealed putative binding energies between the two compounds and CCND1/CDK4/PLK1/CD44 oncogenic signatures. We also showed the antiproliferative and cytotoxic effects of NSC7565600 and NSC765691 against a panel of NCI-60 cancer cell lines. This suggests the potential of NSC765600 and NSC765691 to inhibit CCND1/CDK4/PLK1/CD44 expressions in cancer. Further in vitro and in vivo studies in tumor-bearing mice will be performed to investigate the potential treatment efficacies of the novel NSC765600 and NSC765691 small molecules.

Supplementary Materials: The following are available online at https:/ / www.mdpi.com/article/10 .3390 / cancers13112523/s1, Figure S1: Bioavailability radar showing, six important properties for oral bioavailability, Figure S2: title, Figure S3: Pie charts showing NSC765600 and NSC765691 potential druggable genes.

Author Contributions: N.M. wrote the manuscript; Y.-C.W. oversaw the study and provided funding acquisition; B.L. and H.K. helped with data collection and analyses; H.-S.H. and M.R.S. synthesized and provided NSC765600 and NSC765691; H.-S.H. and A.T.H.W. designed and oversaw the study. All authors have read and agreed to the published version of the manuscript.

Funding: Hsu-Shan Huang was funded by the Ministry of Science and Technology (MOST109-2113M-038-003). Hsu-Shan Huang was funded by Taipei Medical University. Yu-Chi Wang was also funded by the Ministry of Science and Technology (MOST109-2221-E-016-002-MY3).

Data Availability Statement: The datasets generated and/or analyzed in this study are available upon reasonable request.

Acknowledgments: The NCI Developmental Therapeutics Program (DTP) for the 60-cancer-cell line screening of selected compounds described in this paper was funded by the National Cancer Institute, National Institutes of Health (NIH-NCI). We also acknowledge the editing services provided by the Office of Research and Development, Taipei Medical University.

Conflicts of Interest: The authors declare that they have no conflicts of interest. 


\section{References}

1. Christensen, J.F.; Simonsen, C.; Hojman, P. Exercise Training in Cancer Control and Treatment. Compr. Physiol. 2018, 9, 165-205. [CrossRef]

2. Ferlay, J.; Soerjomataram, I.; Dikshit, R.; Eser, S.; Mathers, C.; Rebelo, M.; Parkin, D.M.; Forman, D.; Bray, F. Cancer incidence and mortality worldwide: Sources, methods and major patterns in GLOBOCAN 2012. Int. J. Cancer 2014, 136, E359-E386. [CrossRef]

3. Rizvi, S.M.D.; Alshammari, A.A.A.; Almawkaa, W.A.; Ahmed, A.B.F.; Katamesh, A.; AlAfnan, A.; Almutairi, T.J.; Alshammari, R.F. An oncoinformatics study to predict the inhibitory potential of recent FDA-approved anti-cancer drugs against human Polo-like kinase 1 enzyme: A step towards dual-target cancer medication. 3 Biotech 2019, 9, 70. [CrossRef]

4. O'Donnell, J.S.; Teng, M.W.L.; Smyth, M.J. Cancer immunoediting and resistance to T cell-based immunotherapy. Nat. Rev. Clin. Oncol. 2019, 16, 1511-1567. [CrossRef] [PubMed]

5. Zugazagoitia, J.; Guedes, C.; Ponce, S.; Ferrer, I.; Molina-Pinelo, S.; Paz-Ares, L. Current Challenges in Cancer Treatment. Clin. Ther. 2016, 38, 1551-1566. [CrossRef] [PubMed]

6. Baker, S.J.; Reddy, E.P. CDK4: A Key Player in the Cell Cycle, Development, and Cancer. Genes Cancer 2012, 3, 658-669. [CrossRef] [PubMed]

7. Tchakarska, G.; Sola, B. The double dealing of cyclin D1. Cell Cycle 2020, 19, 163-178. [CrossRef]

8. Malumbres, M.; Barbacid, M. To cycle or not to cycle: A critical decision in cancer. Nat. Rev. Cancer 2001, 1, 222-231. [CrossRef]

9. Musgrove, E.A.; Caldon, C.E.; Barraclough, J.; Stone, A.; Sutherland, R.L. Cyclin D as a therapeutic target in cancer. Nat. Rev. Cancer 2011, 11, 558-572. [CrossRef]

10. The Cancer Genome Atlas Research Network. Erratum: Corrigendum: Comprehensive genomic characterization defines human glioblastoma genes and core pathways. Nat. Cell Biol. 2013, 494, 506. [CrossRef]

11. Cohen, E.E.W.; Soulières, D.; Le Tourneau, C.; Dinis, J.; Licitra, L.; Ahn, M.-J.; Soria, A.; Machiels, J.-P.; Mach, N.; Mehra, R.; et al. Pembrolizumab versus methotrexate, docetaxel, or cetuximab for recurrent or metastatic head-and-neck squamous cell carcinoma (KEYNOTE-040): A randomised, open-label, phase 3 study. Lancet 2019, 393, 156-167. [CrossRef]

12. Chen, Y.; Huang, Y.; Gao, X.; Li, Y.; Lin, J.; Chen, L.; Chang, L.; Chen, G.; Guan, Y.; Pan, L.K.; et al. CCND1 Amplification Contributes to Immunosuppression and is Associated with a Poor Prognosis to Immune Checkpoint Inhibitors in Solid Tumors. Front. Immunol. 2020, 11, 1620. [CrossRef] [PubMed]

13. Chen, J.; Li, X.; Cheng, Q.; Ning, D.; Ma, J.; Zhang, Z.; Chen, X.; Jiang, L. Effects of cyclin D1 gene silencing on cell proliferation, cell cycle, and apoptosis of hepatocellular carcinoma cells. J. Cell. Biochem. 2018, 119, 2368-2380. [CrossRef]

14. Montalto, F.I.; De Amicis, F. Cyclin D1 in Cancer: A Molecular Connection for Cell Cycle Control, Adhesion and Invasion in Tumor and Stroma. Cells 2020, 9, 2648. [CrossRef] [PubMed]

15. Diehl, J.A.; Zindy, F.; Sherr, C.J. Inhibition of cyclin D1 phosphorylation on threonine-286 prevents its rapid degradation via the ubiquitin-proteasome pathway. Genes Dev. 1997, 11, 957-972. [CrossRef]

16. Malumbres, M.; Barbacid, M. Cell cycle, CDKs and cancer: A changing paradigm. Nat. Rev. Cancer 2009, 9, 153-166. [CrossRef] [PubMed]

17. Wang, T.C.; Cardiff, R.D.; Zukerberg, L.; Lees, E.; Arnold, A.; Schmidt, E.V. Mammary hyperplasia and carcinoma in MMTV-cyclin D1 transgenic mice. Nat. Cell Biol. 1994, 369, 669-671. [CrossRef]

18. Van De Weerdt, B.C.; Littler, D.R.; Klompmaker, R.; Huseinovic, A.; Fish, A.; Perrakis, A.; Medema, R.H. Polo-box domains confer target specificity to the Polo-like kinase family. Biochim. Biophys. Acta (BBA) Mol. Cell Res. 2008, 1783, 1015-1022. [CrossRef]

19. Gutteridge, R.E.A.; Ndiaye, M.A.; Liu, X.; Ahmad, N. Plk1 Inhibitors in Cancer Therapy: From Laboratory to Clinics. Mol. Cancer Ther. 2016, 15, 1427-1435. [CrossRef]

20. Wolf, G.; Elez, R.; Doermer, A.; Holtrich, U.; Ackermann, H.; Stutte, H.J.; Altmannsberger, H.-M.; Rübsamen-Waigmann, H.; Strebhardt, K. Prognostic significance of polo-like kinase (PLK) expression in non-small cell lung cancer. Oncogene 1997, 14, 543-549. [CrossRef]

21. Knecht, R.; Elez, R.; Oechler, M.; Solbach, C.; Von Ilberg, C.; Strebhardt, K. Prognostic significance of polo-like kinase (PLK) expression in squamous cell carcinomas of the head and neck. Cancer Res. 1999, 59, 2794-2797.

22. Montaudon, E.; Nikitorowicz-Buniak, J.; Sourd, L.; Morisset, L.; El Botty, R.; Huguet, L.; Dahmani, A.; Painsec, P.; Nemati, F.; Vacher, S.; et al. PLK1 inhibition exhibits strong anti-tumoral activity in CCND1-driven breast cancer metastases with acquired palbociclib resistance. Nat. Commun. 2020, 11, 1-16. [CrossRef] [PubMed]

23. Medina-Aguilar, R.; Marchat, L.A.; Ocampo, E.A.; Gariglio, P.; Mena, J.G.; Sepúlveda, N.V.; Castillo, M.M.; López-Camarillo, C. Resveratrol inhibits cell cycle progression by targeting Aurora kinase A and Polo-like kinase 1 in breast cancer cells. Oncol. Rep. 2016, 35, 3696-3704. [CrossRef] [PubMed]

24. Zhu, L.; Xing, S.; Zhang, L.; Yu, J.-M.; Lin, C.; Yang, W.-J. Involvement of Polo-like kinase 1 (Plk1) in quiescence regulation of cancer stem-like cells of the gastric cancer cell lines. Oncotarget 2017, 8, 37633-37645. [CrossRef] [PubMed]

25. Matthews, H.; Hanison, J.; Nirmalan, N. “Omics"-Informed Drug and Biomarker Discovery: Opportunities, Challenges and Future Perspectives. Proteomes 2016, 4, 28. [CrossRef]

26. Paananen, J.; Fortino, V. An omics perspective on drug target discovery platforms. Brief. Bioinform. 2020, 21, 1937-1953. [CrossRef]

27. Zhong, G.-X.; Hu, J.-Q.; Zhao, K.; Chen, L.-L.; Hu, W.-X.; Qiu, M.-Y. Synthesis and biological evaluation of amide derivatives of diflunisal as potential anti-inflammatory agents. Bioorg. Med. Chem. Lett. 2009, 19, 516-519. [CrossRef]

28. Markham, A. Fostamatinib: First Global Approval. Drugs 2018, 78, 959-963. [CrossRef] 
29. Zhong, G.-X.; Chen, L.-L.; Li, H.-B.; Liu, F.-J.; Hu, J.-Q.; Hu, W.-X. Synthesis and biological evaluation of amide derivatives of diflunisal as potential anti-tumor agents. Bioorg. Med. Chem. Lett. 2009, 19, 4399-4402. [CrossRef]

30. Daina, A.; Michielin, O.; Zoete, V. SwissADME: A free web tool to evaluate pharmacokinetics, drug-likeness and medicinal chemistry friendliness of small molecules. Sci. Rep. 2017, 7, 42717. [CrossRef]

31. Lipinski, C.A. Lead- and drug-like compounds: The rule-of-five revolution. Drug Discov. Today Technol. 2004, 1, 337-341. [CrossRef]

32. Martin, Y.C. A Bioavailability Score. J. Med. Chem. 2005, 48, 3164-3170. [CrossRef]

33. Daina, A.; Zoete, V. A Boiled-Egg to Predict Gastrointestinal Absorption and Brain Penetration of Small Molecules. ChemMedChem 2016, 11, 1117-1121. [CrossRef]

34. Gfeller, D.; Grosdidier, A.; Wirth, M.; Daina, A.; Michielin, O.; Zoete, V. SwissTargetPrediction: A web server for target prediction of bioactive small molecules. Nucleic Acids Res. 2014, 42, W32-W38. [CrossRef] [PubMed]

35. Shoemaker, R.H. The NCI60 human tumour cell line anticancer drug screen. Nat. Rev. Cancer 2006, 6, 813-823. [CrossRef]

36. Hanwell, M.D.; Curtis, D.E.; Lonie, D.C.; Vandermeersch, T.; Zurek, E.; Hutchison, G.R. Avogadro: An advanced semantic chemical editor, visualization, and analysis platform. J. Cheminform. 2012, 4, 17. [CrossRef]

37. Morris, G.M.; Huey, R.; Olson, A.J. Using AutoDock for Ligand-Receptor Docking. Curr. Protoc. Bioinform. 2008, 24, 8.14.1-8.14.40. [CrossRef]

38. Raman, E.P.; Paul, T.J.; Hayes, R.L.; Brooks, I.C.L. Automated, Accurate, and Scalable Relative Protein-Ligand Binding FreeEnergy Calculations Using Lambda Dynamics. J. Chem. Theory Comput. 2020, 16, 7895-7914. [CrossRef] [PubMed]

39. Küçükgüzel, Ş.G.; Küçükgüzel, I.; Tatar, E.; Rollas, S.; Şahin, F.; Güllüce, M.; De Clercq, E.; Kabasakal, L. Synthesis of some novel heterocyclic compounds derived from diflunisal hydrazide as potential anti-infective and anti-inflammatory agents. Eur. J. Med. Chem. 2007, 42, 893-901. [CrossRef]

40. Liechti, C.; Séquin, U.; Bold, G.; Furet, P.; Meyer, T.; Traxler, P. Salicylanilides as inhibitors of the protein tyrosine kinase epidermal growth factor receptor. Eur. J. Med. Chem. 2004, 39, 11-26. [CrossRef] [PubMed]

41. Ritchie, T.J.; Ertl, P.; Lewis, R. The graphical representation of ADME-related molecule properties for medicinal chemists. Drug Discov. Today 2011, 16, 65-72. [CrossRef] [PubMed]

42. Coussens, N.P.; Braisted, J.C.; Peryea, T.; Sittampalam, G.S.; Simeonov, A.; Hall, M.D. Small-Molecule Screens: A Gateway to Cancer Therapeutic Agents with Case Studies of Food and Drug Administration-Approved Drugs. Pharmacol. Rev. 2017, 69, 479-496. [CrossRef]

43. Uhlén, M.; Fagerberg, L.; Hallström, B.M.; Lindskog, C.; Oksvold, P.; Mardinoglu, A.; Sivertsson, Å.; Kampf, C.; Sjöstedt, E.; Asplund, A.; et al. Tissue-based map of the human proteome. Science 2015, 347, 1260419. [CrossRef]

44. Nowell, P.C. The clonal evolution of tumor cell populations. Science 1976, 194, 23-28. [CrossRef] [PubMed]

45. Keller, T.H.; Pichota, A.; Yin, Z. A practical view of 'druggability'. Curr. Opin. Chem. Biol. 2006, 10, 357-361. [CrossRef]

46. Morris, G.M.; Lim-Wilby, M. Molecular Docking. Methods Mol. Biol. 2008, 443, 365-382. [CrossRef]

47. Ma, R.; Ma, Z.-G.; Zhen, C.-L.; Shen, X.; Li, S.-L.; Li, L.; Zheng, Y.-F.; Dong, D.-L.; Sun, Z.-J. Design, synthesis and characterization of poly (methacrylic acid-niclosamide) and its effect on arterial function. Mater. Sci. Eng. C 2017, 77, 352-359. [CrossRef] [PubMed] 